\title{
SPECTRAL ENCLOSURES AND COMPLEX RESONANCES FOR GENERAL SELF-ADJOINT OPERATORS
}

\author{
E.B. DAVIES
}

\begin{abstract}
This paper considers a number of related problems concerning the computation of eigenvalues and complex resonances of a general self-adjoint operator $H$. The feature which ties the different sections together is that one restricts oneself to spectral properties of $H$ which can be proved by using only vectors from a pre-assigned (possibly finite-dimensional) linear subspace $\mathcal{L}$.
\end{abstract}

\section{Introduction}

This paper considers a number of related problems concerning the computation of eigenvalues and complex resonances of a general self-adjoint operator $H$ when one is provided with limited information about the operator. The feature which ties the different sections together is that one restricts oneself to spectral properties of $H$ which can be proved by using only vectors from a pre-assigned (possibly finite-dimensional) linear subspace $\mathcal{L}$ which is not invariant with respect to the operator. Problems of this type arise in numerical analysis and quantum chemistry (and probably elsewhere). We concentrate on the first of these applications, but discuss the second briefly at the end of this section. We also mention some related work on a generalization of the classical Szegó limit theorem in Section 5. While the results of Sections 1 to 4 are reformulations of theorems and techniques used by numerical analysts, the later sections are entirely new to the best of our knowledge, particularly our definition of complex resonances/higher-order spectra in Section 9.

A well-known method for obtaining upper and lower bounds on the eigenvalues of selfadjoint operators depends upon the choice of a parameter $\rho$ and a test-function $f$, followed by the computation of Temple's ratio

$$
\frac{\langle H f, H f\rangle-\rho\langle H f, f\rangle}{\langle H f, f\rangle-\rho\langle f, f\rangle} .
$$

Our first goal is to show how the upper and lower bounds of eigenvalues depend upon the choice of $\rho$ and $f$. The dependence upon $\rho$ appears not to have been previously investigated from our point of view, but our theorems are confirmed by numerical data in earlier publications, as well as by our own computations for a test example. We mention particularly the recent papers of Zimmerman and Mertins [33], [27] who obtained enclosures for eigenvalues in the gap between two parts of the essential spectrum of a self-adjoint operator. This part of our analysis depends heavily upon a theorem of Kato [19] which has been little appreciated outside the numerical analysis community [17]. The novel feature of our approach is that it depends upon the study of a particular non-negative function $F$

I should like to thank W. Arveson, M. Brown, Y. Last, M. Plum, Y. Safarov, A. A. Shkalikov and B. Simon for helpful comments during the research involved in writing this paper.

Received 1st March 1997, revised 3rd February 1998; published 1st June 1998. 1991 Mathematics Subject Classification 49R05, 49R10, 35P15, 47A75, 81Q10

(C) 1998, E.B. Davies 
on the real line, which encapsulates much of the information about the given self-adjoint operator which can be obtained by the use of a given linear subspace $\mathcal{L}$ of the Hilbert space. This function is effectively computable, and its geometrical and analytic properties help to clarify a number of related spectral problems. In particular, it enables one to avoid the spurious eigenvalues which may be obtained if one tries to approximate the spectrum of $H$ by computing the spectrum of $P H P$, where $P$ is the orthogonal projection onto the subspace $\mathcal{L}$. Our main results concerning the discrete spectrum are Theorems 3 and 8 , but their relevance only becomes clear from the surrounding comments concerning their numerical application.

In Section 6 we consider the determination of the essential spectrum of discretised Schrödinger operators with bounded potentials. Arveson [4], [5], [3] has recently used $C^{*}$ algebra methods in conjunction with a computer program to investigate the complicated spectra possible for the almost Mathieu equation, a one-dimensional model with an almost periodic potential. The methods of the present paper throw further light on the nature of the spurious eigenvalues, and indicate how to eliminate them before passing to the infinite volume limit.

In Section 7 we describe an adaptation to Schrödinger operators of a theorem of Avron, van Mouche and Simon [6] which enables one to locate the whole spectrum of such an operator approximately, by considering only test functions which are supported in balls of given radius. Although not particularly efficient, it is suprising that such bounds may be proved witout any local or global restrictions on the potential, apart from the requirement that it be non-negative.

Finally, in Sections 8 and 9, we consider the problem from a more theoretical point of view, which leads to a new definition of the concept of resonance of an arbitrary self-adjoint operator relative to a given subspace. This definition is purely functionally analytical, and does not depend upon the operator in question having a non-empty absolutely continuous spectrum, as do all other definitions of resonance that we know of. We define a hierarchy of $n$-th order spectra of $H$ with respect to a subspace, and show that there is a very close relationship between the function $F$ previously considered and the second-order spectrum, which is usually entirely non-real. We also provide explicit computations for a simple example.

When studying the discrete spectrum, we consider only the simplest case, namely finding rigorous enclosures for solutions of $H f=\lambda f$, where $H$ is a possibly unbounded selfadjoint operator acting in a Hilbert space $\mathscr{H}$. The methods can be extended to the equation $A f=\lambda B f$ where one of the self-adjoint operators $A, B$ is positive definite. There are two standard and complementary methods of bounding the eigenvalues of a self-adjoint operator $H$, associated with Rayleigh-Ritz and Temple-Lehmann-Goerisch. Both methods involve choosing a finite-dimensional subspace of the domain of the operator, followed by the implementation of certain matrix calculations. We focus on three aspects of the procedures.

The first is the choice of the finite-dimensional subspace $\mathcal{L}$ of the Hilbert space $\mathcal{H}$. This may well be the most important issue of all. If $\mathcal{L}$ is even approximately orthogonal to some eigenvector of $H$, then no computations involving the restriction of $H$ to $\mathcal{L}$ can possibly allow one to get a good approximation to the associated eigenvalue. Even if this radical failure does not occur, the quality of any estimate depends critically upon how well the subspace is adapted to the operator $H$. Finite element subspaces provide a reasonable general choice for many partial differential operators, but for particular problems of this type one might do much better by choosing a basis of functions which have the appropriate behaviour at local singularities. Another interesting possibility, discussed in Section 5, is 
to choose $\mathcal{L}$ to be a spectral subspace of an auxiliary operator. The quality of the spectral information obtained can then be estimated in terms of certain commutators involving the two operators.

The second issue is that of rounding errors in the numerical calculations. Interval arithmetic methods give guaranteed bounds, but are slower to implement. The recommended method is usually to obtain the best possible approximations to the eigenvalue and eigenvector using standard floating-point arithmetic, and then to start again, using these approximations, to obtain rigorous upper and lower bounds by means of a spectral inequality together with interval arithmetic. This last stage should involve as few calculations as possible.

The third issue, and the one which we concentrate on, is that of the spectral inequalities themselves. The Rayleigh-Ritz procedure provides upper bounds for those eigenvalues of $H$ which are less than the bottom of the essential spectrum. Complementary lower bounds have been obtained by Temple, Lehmann and Goerisch; see [13], [28], [27], [33] for accounts of these methods. However the methods do not work as they stand for eigenvalues in a spectral gap. Our goal in this paper is to reinvestigate this issue and to provide a new perspective on the Temple-Lehmann procedure, which may even be of computational value.

We assume that $\mathcal{L}$ is a linear subspace of the domain of the self-adjoint operator $H$, and that $A=\left.H\right|_{\mathscr{L}}$ is a closed operator; this is automatic if $\mathcal{L}$ is finite-dimensional. Let $P$ be the orthogonal projection onto the closure $\overline{\mathcal{L}}$ of $\mathcal{L}$, and let $B=P A$. Then $B$ is a symmetric operator acting in the Hilbert space $\overline{\mathcal{L}}$. If $\mathcal{L}$ is finite-dimensional then $B$ is self-adjoint, but in general this is not the case. A standard procedure for calculating approximate eigenvalues of $H$ is to calculate the eigenvalues of $B$, or of some particular self-adjoint extension of $B$ in $\overline{\mathcal{L}}$. However, this may lead to serious errors: if $H$ is a differential operator, the choice of $\mathcal{L}$ may be associated with boundary conditions which lead to spurious eigenvalues, the eigenfunctions of which are concentrated near to the boundary [14, Lemma 6]. Whether it is easy to identify these spurious eigenvalues may depend on the way in which $\mathcal{L}$ is defined. We refer to [4], [32, p. 201] for some famous examples for which the spectrum of $B$ and of the original operator $H$ are totally unrelated in the limit as $\mathcal{L}$ increases towards $\mathcal{H}$.

We conclude the section by explaining the relevance of the theory presented here to quantum chemistry. There are standard prescriptions for calculating the energy levels of molecules (or atoms), but these assume that the molecule is isolated in space. For a typical molecule, surrounded by others and by stray electric fields, it is of interest to ask about those spectral properties which are stable with respect to a range of possibly random perturbations of the potential outside the immediate neighbourhood of the molecule. One way of posing this question is by considering the usual Hamiltonian but confining oneself to information which can be obtained by the use of wave-functions which have support in a defined neighbourhood of the nuclei. Our procedure does not amount to imposing Dirichlet or any other particular boundary conditions on the operator in the region considered. If one chooses the subspace $\mathcal{L}$ using this prescription, our method provides a procedure for defining and computing spectral properties, including complex resonances.

\section{The basic method}

We base our analysis upon the behaviour of the non-negative real-valued function

$$
F(t)=\inf \left\{\frac{\|A \phi-t \phi\|}{\|\phi\|}: 0 \neq \phi \in \mathcal{L}\right\}
$$


and higher-order analogues $F_{n}(t)$ for $n \geqslant 2$. Note that if $\mathcal{L}$ is finite-dimensional then $F(t)=0$ if and only if $t$ is an eigenvalue of $H$ whose eigenfunction lies in $\mathcal{L}$. This unlikely possibility need not concern us any further.

The connection between the function $F(t)$ and the Temple-Lehmann-Goerisch formula for the enclosure of eigenvalues is not obvious, and we refer the reader who wishes to see this explained immediately to Theorem 6 .

Lemma 1. The function $t \rightarrow F(t)$ is Lipschitz continuous and satisfies

$$
|F(s)-F(t)| \leqslant|s-t|
$$

for all $s, t \in \mathbf{R}$ and also

$$
F(t) \geqslant d_{H}(t):=\operatorname{dist}(t, \operatorname{Spec}(H)) .
$$

Proof. Given $0 \neq \phi \in \mathcal{L}$, we have

$$
F(t) \leqslant \frac{\|A \phi-t \phi\|}{\|\phi\|} \leqslant \frac{\|A \phi-s \phi\|}{\|\phi\|}+|s-t| .
$$

Taking the inf over all such $\phi$ leads quickly to the first inequality. If $t \notin \operatorname{Spec}(H), \phi$ is as before and $\psi:=A \phi-t \phi$, then

$$
\frac{\|\phi\|}{\|A \phi-t \phi\|}=\frac{\left\|(H-t I)^{-1} \psi\right\|}{\|\psi\|} \leqslant\left\|(H-t I)^{-1}\right\|=d_{H}(t)^{-1}
$$

so

$$
d_{H}(t) \leqslant \frac{\|A \phi-t \phi\|}{\|\phi\|}
$$

and we may now take the inf over all $\phi$ to obtain the second inequality. If $t \in \operatorname{Spec}(H)$ then the second inequality is elementary.

It is found in a number of test cases that $F$ and $d_{H}$ are very similar even when the subspace $\mathcal{L}$ has quite low dimension. Thus any local minimum of $F(t)$ is close to the spectrum of $H$, provided the value $F(t)$ is small, an observation due to Krylov and Weinstein [7, Cor. 6.20] :

Corollary 2. If $0 \leqslant F(t) \leqslant \delta$, then

$$
\operatorname{Spec}(H) \cap[t-\delta, t+\delta] \neq \emptyset,
$$

for any self-adjoint extension $H$ of the operator $A$ on $\mathcal{L}$.

This corollary will be reconsidered in Section 5, when we turn to the study of the essential spectrum of $H$. Until that section we assume that the essential spectrum is known, and that we are interested in determining eigenvalues in the gaps between parts of the essential spectrum as accurately as possible.

Corollary 2 yields a reasonable first estimate of the eigenvalues of $H$, but it is typically much less efficient than the method of Theorem 3 below. It does not, and cannot, disprove the possibility that $F(s)$ could be large even when $s \in \operatorname{Spec}(H)$. (See the comments made in Section 1 about the importance of a good selection of $\mathcal{L}$.) Thus the use of the function $F$ might not help to locate all of the eigenvalues of $H$, but it cannot lead to the "discovery" of spurious eigenvalues. The following typical example of a function $F(s)$, shown in Figure 1, was produced using [9]. 


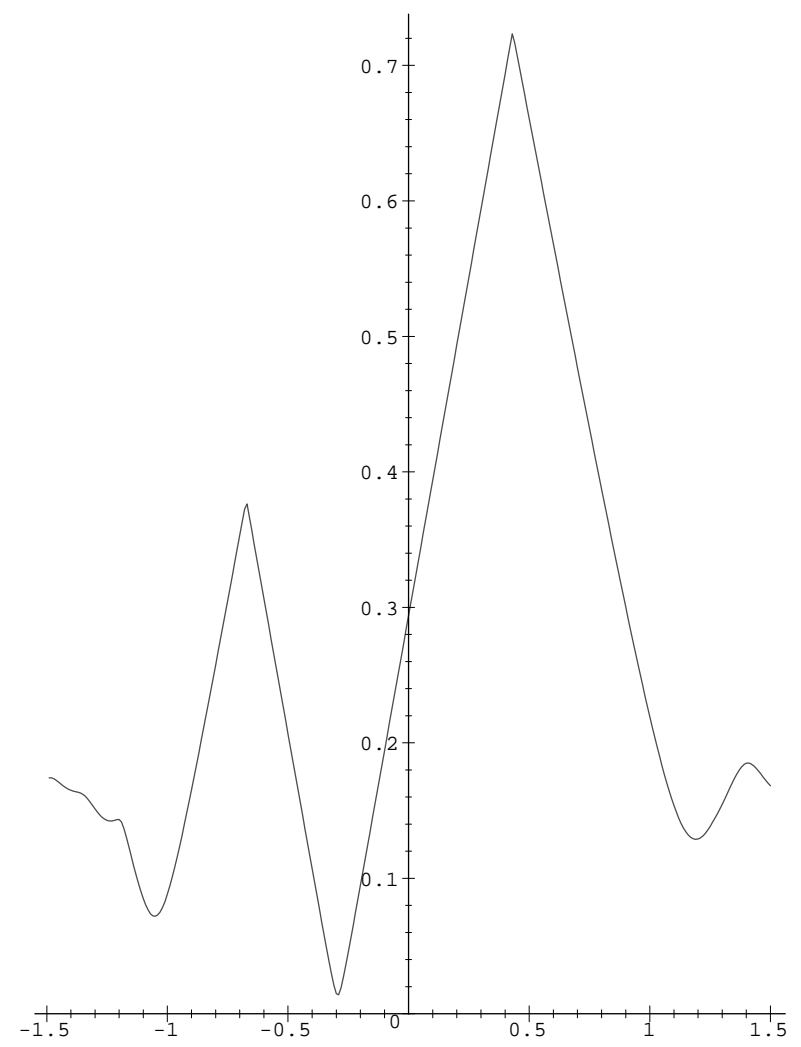

Figure 1: A function $F(s)$, produced using [9]. 
It is surprising that one gets much better enclosures of the eigenvalues of $H$ by evaluating $F(s)$ near its local maxima rather than near its local minima. The best theoretical choices of $s$ are given in a standard context by the following theorem. We assume that $H$ has exactly one simple eigenvalue $\lambda$ in the interval $\left(\rho^{-}, \rho^{+}\right)$. Elaborations of the theorem are presented later. The assumptions of the theorem become easier to satisfy as $\rho^{-}$decreases and $\rho^{+}$ increases, so the optimal choices are

$$
\begin{aligned}
& \rho^{-}=\max \{s \in \operatorname{Spec}(H): s<\lambda\} \\
& \rho^{+}=\min \{s \in \operatorname{Spec}(H): s>\lambda\} .
\end{aligned}
$$

If $\lambda$ is the smallest (respectively, the largest) point in the spectrum of $H$, then the optimal choices are $\rho^{-}=-\infty$ (respectively, $\left.\rho^{+}=+\infty\right)$.

The amount of improvement obtained by the use of Theorems 3 or 4 to locate $\operatorname{Spec}(H)$, as opposed to Corollary 2, is impressive. We refer to Section 4 for a numerical example, and for other relevant computations in the literature.

Theorem 3. Let $\rho^{-}<\sigma^{-}<\sigma^{+}<\rho^{+}$, and let $\lambda$ be the only point of the spectrum of $H$ in $\left(\rho^{-}, \rho^{+}\right)$. If

$$
F\left(\sigma^{-}\right)<\sigma^{-}-\rho^{-}, \quad F\left(\sigma^{+}\right)<\rho^{+}-\sigma^{+} .
$$

then

$$
\sigma^{+}-F\left(\sigma^{+}\right) \leqslant \lambda \leqslant \sigma^{-}+F\left(\sigma^{-}\right) .
$$

If one assumes in addition that $\rho^{ \pm} \notin \operatorname{Spec}(H)$, then (2.6) also follows from

$$
F\left(\sigma^{-}\right)=\sigma^{-}-\rho^{-}, \quad F\left(\sigma^{+}\right)=\rho^{+}-\sigma^{+} .
$$

Proof. By Corollary 2 there is a point of $\operatorname{Spec}(H)$ in $\left[\sigma^{+}-F\left(\sigma^{+}\right), \sigma^{+}+F\left(\sigma^{+}\right)\right]$and this forces $\sigma^{+}-F\left(\sigma^{+}\right) \leqslant \lambda$. The other inequality has a similar proof, while the second version of the theorem is an elementary consequence.

The following theorem clarifies the principles underlying the best choice of $\sigma^{ \pm}$.

Theorem 4. Let

$$
\rho^{-}<\sigma_{1}^{-}<\sigma_{2}^{-}<\sigma_{2}^{+}<\sigma_{1}^{+}<\rho^{+}
$$

and

$$
F\left(\sigma_{i}^{-}\right)<\sigma_{i}^{-}-\rho^{-}, \quad F\left(\sigma_{i}^{+}\right)<\rho^{+}-\sigma_{i}^{+}
$$

for $i=1,2$. Then

$$
\sigma_{2}^{+}-F\left(\sigma_{2}^{+}\right) \leqslant \sigma_{1}^{+}-F\left(\sigma_{1}^{+}\right) \leqslant \lambda \leqslant \sigma_{1}^{-}+F\left(\sigma_{1}^{-}\right) \leqslant \sigma_{2}^{-}+F\left(\sigma_{2}^{-}\right) .
$$

Proof. The inner two inequalities are taken from Theorem 3, and the outer two from the first half of Lemma 1.

The following comments are in order.

1) Theorem 4 may be summarised by saying that $\sigma^{ \pm}$should be as far away from $\lambda$ as possible, subject to the stated constraints, in order to obtain the best enclosure of $\lambda$.

2) It is clear from these theorems and Figure 1 that if we knew the exact values of $\lambda, \rho^{+}, \rho^{-}$, and could compute $F$ without error then by solving the equations (2.7) we would obtain the optimal choices of $\sigma^{ \pm}$for the enclosures (2.6). The problem is how to proceed in the absence of this information. 
3) The theorems may be implemented in practice as follows. One first obtains approximate but unverified values of $\lambda, \rho^{+}, \rho^{-}$. If $F$ is very close to $d_{H}$ then one should put $\sigma^{+} \doteq\left(\lambda+\rho^{+}\right) / 2$ and $\sigma^{-} \doteq\left(\lambda+\rho^{-}\right) / 2$, that is near the local maxima of $F$. If one is confident that $F$ is close to $d_{H}$ and that one has values of $\lambda, \rho^{+}, \rho^{-}$which are close to the true values, this suggests that one could try

$$
\begin{aligned}
& \sigma^{+}=0.52 \lambda+0.48 \rho^{+} \\
& \sigma^{-}=0.52 \lambda+0.48 \rho^{-} .
\end{aligned}
$$

The acceptability of these choices is determined not by one's confidence in the initial estimates of $\lambda, \rho^{+}$and $\rho^{-}$, but solely by whether the required inequalities (2.5) hold.

4) The function $F$ only has to be evaluated at the chosen points $\sigma^{ \pm}$. Theorem 5 shows that $F\left(\sigma^{ \pm}\right)$is the square root of a certain eigenvalue. There are of course standard packages for evaluating eigenvalues of large matrices, but we need a verified upper bound. The procedure for obtaining this is to use floating-point arithmetic to obtain a good approximation to the eigenvalue and eigenvector. One then starts again using this approximate eigenvector, the Rayleigh-Ritz procedure and interval arithmetic to obtain a verified upper bound on the eigenvalue. Although we only end up with an upper bound on $F\left(\sigma^{ \pm}\right)$, this still yields an enclosure of $\lambda$, albeit a slightly weaker one.

We next describe the numerical procedure for evaluating $F(s)$ for any $s \in \mathbf{R}$, under the assumption that $\mathcal{L}$ is finite-dimensional. We define $N(s): \mathcal{L} \rightarrow \mathcal{L}$ for any $s \in \mathbf{R}$ by

$$
\begin{aligned}
N(s) & :=A^{*} A-2 s B+s^{2} I_{\mathcal{L}} \\
& =C+\left(B-s I_{\mathcal{L}}\right)^{2}
\end{aligned}
$$

where $0 \leqslant C=A^{*}(1-P) A$. The size of the operator $C$ measures the failure of $\mathcal{L}$ to be a spectral subspace of $H$. Let $\lambda_{n}(s), 1 \leqslant n \leqslant \operatorname{dim}(\mathcal{L})$, be the eigenvalues of $N(s)$ written in increasing order and repeated according to multiplicity. They may be computed using standard packages.

Theorem 5. We have

$$
F(s)=\lambda_{1}(s)^{1 / 2}
$$

for all $s \in \mathbf{R}$. There exists an $s$-dependent relabelling $\tilde{\lambda}_{n}(s)$ of the eigenvalues such that $\tilde{\lambda}_{n}(s)$ are all real-analytic functions of $s$.

Proof. The first statement follows from the identity

$$
\langle N(s) \phi, \phi\rangle=\|H \phi-s \phi\|^{2}
$$

for all $\phi \in \mathcal{L}$. The second is a standard consequence of the fact that the operators $N(s)$ form a self-adjoint holomorphic family in the sense of Kato [20].

We now prove the equivalence between our method and that based upon the TempleLehmann-Kato bounds, which is the best general method for obtaining rigorous enclosures of eigenvalues of partial differential operators if one uses a fixed test function space. In contrast to our previous practice, the parameter $\sigma$ is defined in terms of $\rho^{+}, H$ and $\phi$, rather than being an independent variable.

The following theorem has an obvious analogue giving an upper bound on $\lambda$. 
Theorem 6. Let $\lambda<\rho^{+}$and $\left[\lambda, \rho^{+}\right] \cap \operatorname{Spec}(H)=\{\lambda\}$. Let $\phi \in \operatorname{Dom}(H)$ and

$$
\langle H \phi, \phi\rangle<\rho^{+}\|\phi\|^{2} \text {. }
$$

If we put

$$
\sigma=\frac{1}{2} \frac{\left(\rho^{+}\right)^{2}\|\phi\|^{2}-\|H \phi\|^{2}}{\rho^{+}\|\phi\|^{2}-\langle H \phi, \phi\rangle}
$$

then

$$
\rho^{+}-\sigma=\frac{\|H \phi-\sigma \phi\|}{\|\phi\|}=\frac{1}{2} \frac{\left\|\rho^{+} \phi-H \phi\right\|^{2}}{\rho^{+}\|\phi\|^{2}-\langle H \phi, \phi\rangle}>0
$$

and

$$
\lambda \geqslant 2 \sigma-\rho^{+}=\frac{\rho^{+}\langle H \phi, \phi\rangle-\|H \phi\|^{2}}{\rho^{+}\|\phi\|^{2}-\langle H \phi, \phi\rangle} .
$$

Proof. The identity

$$
\rho^{+}-\sigma=\frac{1}{2} \frac{\left\|\rho^{+} \phi-H \phi\right\|^{2}}{\rho^{+}\|\phi\|^{2}-\langle H \phi, \phi\rangle}
$$

is obtained by simple algebra, and implies $\sigma<\rho^{+}$. Hence the equality

$$
\rho^{+}-\sigma=\frac{\|H \phi-\sigma \phi\|}{\|\phi\|}
$$

is equivalent to

$$
\left(\rho^{+}-\sigma\right)^{2}\|\phi\|^{2}=\|H \phi-\sigma \phi\|^{2}
$$

which is again confirmed by simple algebra. We then have

$$
\lambda \geqslant \sigma-F(\sigma) \geqslant 2 \sigma-\rho^{+}
$$

and the final equality is again simple algebra.

Note 1. The lower bound $\lambda \geqslant 2 \sigma-\rho^{+}$is improved by increasing $\sigma$, subject to the existence of a suitable function $\phi$. Assuming that $\phi \in \mathcal{L}$, the optimal situation is obtained by taking $\sigma$ to be the solution of $\rho^{+}-\sigma=F(\sigma)$.

Note 2. If we increase $\rho^{+}$subject to $\left[\lambda, \rho^{+}\right] \cap \operatorname{Spec}(H)=\{\lambda\}$ then by differentiating the Temple ratio we see that we get a better lower bound on $\lambda$. The optimal choice therefore arises by taking $\rho^{+}$to be close to the first spectral point of $H$ to the right of $\lambda$, if this point is known.

\section{An elaboration of the method}

It appears from recent papers that one may sometimes obtain a better enclosure of $\lambda$ by taking $\rho^{+}\left(\rho^{-}\right)$substantially larger (smaller) than suggested above. We describe the theory behind this extension, which once again eventually yields the Temple-LehmannKato bounds.

We define $d_{n, H}(s)$ to be the $n$th eigenvalue of $|H-s I|$ counting in increasing order, repeating according to multiplicity, and stopping at the smallest point of the essential spectrum of $|H-s I|$. This may informally be described as the distance from $s$ to the $n$th eigenvalue of $H$ counting outwards from $s$. It is immediate from the definition that $d_{n, H}(s)$ 
is a continuous, piecewise linear function of $s$ with gradient \pm 1 at every non-exceptional point. A typical graph of $d_{2, H}(s)$ is shown in Figure 2.

We now recall that $\lambda_{n}(s)$ was defined to be the $n$th eigenvalue of $N(s)$.

Theorem 7. We have

$$
d_{n, H}(s) \leqslant F_{n}(s)=\lambda_{n}(s)^{1 / 2}
$$

for all $n$ and $s$, and

$$
\left|F_{n}(s)-F_{n}(t)\right| \leqslant|s-t|
$$

for all $n, s$ and $t$.

Proof. The first inequality follows from the Rayleigh-Ritz method applied to $(H-s I)^{2}$. The second inequality follows from the alternative definition

$$
F_{n}(s)=\min _{L \in \mathcal{E}_{n}} \max \left\{\frac{\|A \phi-s \phi\|}{\|\phi\|}: 0 \neq \phi \in L\right\}
$$

where $\mathcal{E}_{n}$ is the set of all $n$-dimensional subspaces of $\mathcal{L}$.

The same method as that of the last section now yields the following.

Theorem 8. If $\lambda_{n}$ is the $n$th eigenvalue of $H$ counting to the left of $\rho^{+}$, and $\sigma^{+}$satisfies

$$
F_{n}\left(\sigma^{+}\right)<\rho^{+}-\sigma^{+}
$$

then

$$
\lambda_{n} \geqslant \sigma^{+}-F_{n}\left(\sigma^{+}\right)
$$

The implementation of this method is similar to that for the case $n=1$, described after Theorem 4 . The first stage is to use unverified estimates of the eigenvalues to select appropriate values of $\sigma^{ \pm}$. Specifically, we take $\sigma^{+}$as close to $\left(\lambda_{n}+\rho^{+}\right) / 2$ as possible while satisfying

$$
F_{n}\left(\sigma^{+}\right)<\rho^{+}-\sigma^{+}
$$

We then take $\phi$ to be the eigenvector of $N\left(\sigma^{+}\right)$corresponding to its $n$th eigenvalue, and obtain

$$
\lambda_{n} \geqslant \frac{\langle H \phi, H \phi\rangle-\rho^{+}\langle H \phi, \phi\rangle}{\langle H \phi, \phi\rangle-\rho^{+}\langle\phi, \phi\rangle} .
$$

The best choice of $n$ to take depends on circumstances. If $H$ has several very close eigenvalues surrounded by a large gap, then it is almost certainly necessary to take a large value of $n$ to obtain good estimates on the middle eigenvalues of the group. Numerical examples indicate that, even if the eigenvalues are well separated, there are often advantages in choosing $n>1$. See [33, Tables 1 and 2]. The advantage of taking $\rho^{ \pm}$further away from $\lambda$ outweighs the disadvantage of having to compute higher eigenvectors of the operator $N(s)$ for appropriate $s$.

\section{A numerical example}

We illustrate the above theory by means of a simple example which is deliberately computed to lower accuracy than possible so as to make the errors involved in the choice 


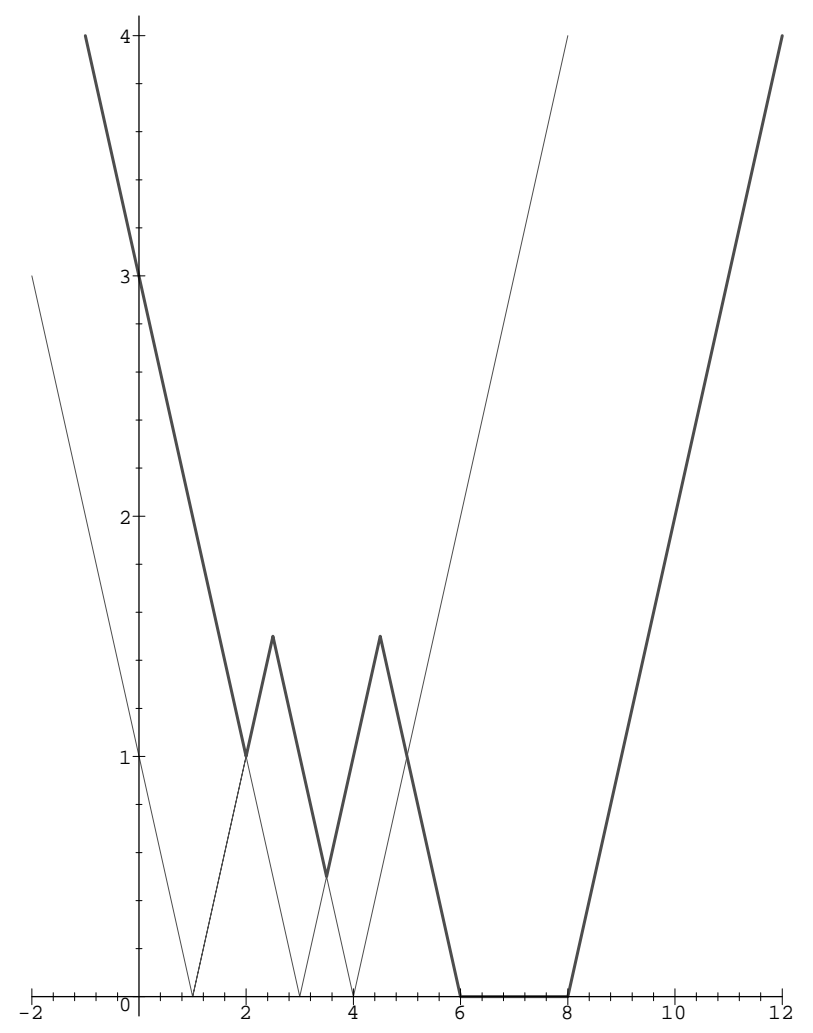

Figure 2: A typical graph of $d_{2, H}(s)$. 
Table 1:

\begin{tabular}{cccc}
\hline$s$ & $F(s)$ & $s+F(s)$ & $s-F(s)$ \\
\hline-.35 & .05788448146 & -.2921155185 & -.4078844815 \\
-.34 & .04821723590 & -.2917827641 & -.3882172359 \\
-.33 & .03871864769 & -.2912813523 & -.3687186477 \\
-.32 & .02955183526 & -.2904481647 & -.3495518353 \\
-.31 & .02115267820 & -.2888473218 & -.3311526782 \\
-.30 & .01488321555 & -.2851167845 & -.3148832156 \\
-.29 & .01398340816 & -.2760165918 & -.3039834082 \\
-.28 & .01922275402 & -.2607772460 & -.2992227540 \\
-.27 & .02726623140 & -.2427337686 & -.2972662314 \\
-.26 & .03629513011 & -.2237048699 & -.2962951301 \\
-.25 & .04572945345 & -.2042705466 & -.2957294535 \\
\hline
\end{tabular}

of $s$ obvious. The operator $H^{0}$ is defined on $l^{2}(\mathbf{Z})$ by the matrix

$$
H_{m n}^{0}=\left\{\begin{array}{cl}
1 & \text { if }|m-n|=1 \\
(-1)^{n} & \text { if } m=n \\
0 & \text { otherwise. }
\end{array}\right.
$$

Fourier transform calculations show that $H^{0}$ is bounded with spectrum $[-\sqrt{5},-1] \cup[1, \sqrt{5}]$. We now define $H$ by

$$
H_{m n}=\left\{\begin{array}{cl}
-2 & \text { if } m=n=k \\
H_{m n}^{0} & \text { otherwise }
\end{array}\right.
$$

for some positive even integer $k$. Since $H$ is a rank one perturbation of $H_{0}$, it has the same essential spectrum, and at most one eigenvalue in each of the gaps of the spectrum of $H_{0}$. In fact, $H_{0}$ has two eigenvalues

$$
\begin{aligned}
& \mu \doteq-3.099952 \\
& \lambda \doteq-0.293684 .
\end{aligned}
$$

We now define $\mathcal{L}$ to be the subspace of sequences $f \in l^{2}(\mathbf{Z})$ with support in $\{1, \ldots, 2 k\}$, and concentrate on obtaining enclosures of $\lambda$ using this subspace only.

We put $k=10$ and compute $F(s), s+F(s), s-F(s)$ for a range of values of $s$ in Table 1 , taken from [9].

If we simply minimise $F(s)$ by putting $s=-0.29$, then we obtain the very poor enclosure

$$
-0.3040 \leqslant \lambda \leqslant-0.2760 \text {. }
$$

Our theorems state that $s-F(s)<\lambda$ provided that $s+F(s)<1$, and that $s+F(s)>\lambda$ provided that $s-F(s)>-1$. We see that the best enclosures of $\lambda$ in Table 2 are obtained by taking $s=-0.64$ and $s=0.35$, the result being

$$
-0.293775 \leqslant \lambda \leqslant-0.293528 \text {. }
$$

This initial enclosure leads us to new values for $s$, namely $\frac{\lambda-1}{2} \doteq-0.646$ and $\frac{\lambda+1}{2} \doteq$ 0.353 . Having confirmed the relevant inequalities for these two values of $s$, we then obtain 
Table 2:

\begin{tabular}{cccc}
\hline$s$ & $F(s)$ & $s+F(s)$ & $s-F(s)$ \\
\hline-.70 & .3532050530 & -.3467949469 & -1.053205053 \\
-.69 & .3628673504 & -.3271326496 & -1.052867350 \\
-.68 & .3725232840 & -.3074767161 & -1.052523284 \\
-.67 & .3763394573 & -.2936605426 & -1.046339457 \\
-.66 & .3664305129 & -.2935694871 & -1.026430513 \\
-.65 & .3564543397 & -.2935456604 & -1.006454340 \\
-.64 & .3464716983 & -.2935283016 & -.9864716982 \\
-.63 & .3364870385 & -.2935129615 & -.9664870385 \\
-.62 & .3265015554 & -.2934984447 & -.9465015555 \\
-.61 & .3165157582 & -.2934842417 & -.9265157581 \\
-.60 & .3065299342 & -.2934700658 & -.9065299342 \\
\hline .30 & .5937971136 & .8937971136 & -.2937971136 \\
.31 & .6037928541 & .9137928541 & -.2937928541 \\
.32 & .6137885136 & .9337885136 & -.2937885136 \\
.33 & .6237840484 & .9537840484 & -.2937840484 \\
.34 & .6337794005 & .9737794005 & -.2937794005 \\
.35 & .6437744895 & .9937744895 & -.2937744895 \\
.36 & .6537691985 & 1.013769199 & -.2937691985 \\
.37 & .6637633485 & 1.033763349 & -.2937633485 \\
.38 & .6737566457 & 1.053756646 & -.2937566457 \\
.39 & .6837485619 & 1.073748562 & -.2937485619 \\
.40 & .6937380144 & 1.093738014 & -.2937380144 \\
\hline
\end{tabular}

the marginally stronger enclosure

$$
-0.293773 \leqslant \lambda \leqslant-0.293538
$$

which depends only upon the evaluation of $F(s)$ at these two points and the fact that $\lambda$ is the only spectral point of $H$ in $(-1,1)$. One could improve this further, but there are obvious limits to what can be achieved with $k=10$. There is no difficulty in taking larger values of $k$, but the purpose of this example is to illustrate the methods. To obtain maximum accuracy, interval arithmetic, which we have not used, would in any case be necessary.

\section{Szegö's theorem}

We mention another approach to the spectral theory of a self-adjoint operator $H$, which depends upon comparing the spectrum of $H$ with that of $P H P$, where $P$ is the orthogonal projection on a given closed subspace $\mathcal{L}$.

The Berezin-Lieb formula

$$
\operatorname{tr}[P \phi(P H P) P] \leqslant \operatorname{tr}[P \phi(H) P],
$$

valid for all convex $\phi$, has recently been generalized by Laptev and Safarov [22], who obtained an estimate for the difference of the two expressions above for a certain class 
of non-convex $\phi$. They used their new formula in [23] to provide a generalization of the classical Szegô limit theorem, described below.

Let $P_{\lambda}$ be the spectral projections of an auxiliary self-adjoint operator $A$, so that $P_{\lambda}$ converge strongly to the identity operator as $\lambda \rightarrow \infty$. If $\psi$ is a suitably smooth function on $\mathbf{R}$ with $\psi(0)=0$, then one may investigate the asymptotics as $\lambda \rightarrow \infty$ of the quantity

$$
\operatorname{tr}\left[\psi\left(P_{\lambda} H P_{\lambda}\right)\right]
$$

Laptev and Safarov [23] obtained an asymptotic formula for this with an explicit error estimate, generalizing Szegő's theorem, and provided several applications to pseudodifferential operators. They assumed that the operators $A$ and $H$ satisfy certain commutator estimates, in order to obtain corresponding commutator estimates for $P$ and $H$. Such commutator estimates are also relevant to the theory of this paper, as we now demonstrate.

If one puts $X:=(I-P) H P$ then it is easy to verify that

$$
P(H-s I)^{2} P=C+(P H P-s P)^{2}
$$

where $C:=X^{*} X$.

A second easy calculation shows that

$$
[P, H]^{*}[P, H]=X^{*} X+X X^{*} .
$$

But it is well known that $X^{*} X$ and $X X^{*}$ have the same spectrum, apart possibly from 0 , so

$$
\|C\|=\|[P, H]\|^{2}
$$

with corresponding results for other $\mathcal{C}_{p}$ norms. For Theorem 10 below, the rank of $C$ is a more important measure of its influence than any of these norms.

It should be emphasised that in Sections 2 and 3 we need no assumptions about the magnitude of the commutator to justify the spectral enclosures obtained. While there is no a priori guarantee that the enclosures are tight, they are guaranteed to be valid, and their accuracy in applications probably exceeds what could be proved by any general prior argument.

\section{The essential spectrum}

Our next theorem states that if one has an appropriate increasing sequence of subspaces $\mathcal{L}_{n}$, then it is possible to determine the spectrum of $H$ exactly using our earlier theorems.

Theorem 9. Suppose $\left\{\mathcal{L}_{n}\right\}_{n=1}^{\infty}$ is an increasing sequence of closed subspaces of $\mathscr{H}$ such that $\mathscr{D}:=\cup_{n=1}^{\infty}\left\{\mathcal{L}_{n} \cap \operatorname{Dom}(H)\right\}$ is a core for the self-adjoint operator $H$. If $F^{(n)}$ are the functions associated with $\mathcal{L}_{n}$ according to $(2.1)$ then $F^{(n)}$ decrease monotonically and converge locally uniformly to $d_{H}$. In particular, $s \in \operatorname{Spec}(H)$ if and only if

$$
\lim _{n \rightarrow \infty} F^{(n)}(s)=0 .
$$

Note. While this confirms the theoretical importance of the functions $F^{(n)}$ for the spectral theory of $H$, it can only be of use numerically if supplemented by information about the rate of convergence.

If we consider $H:=-\Delta+V(x)$ acting in $L^{2}\left(\mathbf{R}^{N}\right)$ and take $\mathcal{L}_{n}$ to be the space of functions with support in $\{x:|x| \leqslant n\}$, then the hypothesis of the theorem is satisfied if for example $V$ is bounded below and it is locally $L^{2}$. If $H$ has a bounded (non- $L^{2}$ ) eigenfunction 
associated with the value $s \in \mathbf{R}$, then one might expect that $F^{(n)}(s)=O\left(\frac{1}{n}\right)$. The precision of this method does not compare with that for computations of discrete eigenvalues.

Since the essential spectrum, and in particular the continuous spectrum of a Schrödinger operator, depend entirely upon the asymptotic behaviour of the potential at infinity, its location is best determined by using theorems about the essential spectrum if possible. If no such theorems are available for a particular operator, then one may be forced to the type of procedure which we now describe.

The method of Arveson [4], [5] for determining the essential spectrum has some relationship with our analysis, but does not involve the functions $F(s)$, which we regard as central. He invokes $C^{*}$-algebra theory to deal with some discretised problems in one dimension, and produces efficient algorithms in that context. However, his paper only discusses the infinite volume limit, without controlling the rate of convergence, while we are interested below in obtaining guaranteed bounds using one chosen subspace. We consider only discretised Schrödinger operators for definiteness, but the same methods can be applied to partial differential operators.

Let $\mathscr{H}:=l^{2}\left(\mathbf{Z}^{N}\right)$, and let $\mathcal{L}$ be the finite-dimensional subspace of $\mathscr{H}$ consisting of functions with support in some large finite subset $S$ of $\mathbf{Z}^{N}$. Let $H:=H_{0}+V$ where $V$ is a real-valued bounded potential on $\mathbf{Z}^{N}$ and

$$
H_{0} f(n):=\sum_{m \sim n} f(m)
$$

where $m \sim n$ if $\sum_{i=1}^{N}\left|m_{i}-n_{i}\right|=1$. It is immediate that $\|H\| \leqslant c:=2 N+\|V\|_{\infty}$. Our task is to obtain as much information about $\operatorname{Spec}(H) \subseteq[-c, c]$ as possible from within the subspace $\mathcal{L}$. We must avoid spurious eigenvalues associated with eigenfunctions which are concentrated around the boundary.

$$
\partial S:=\{n \notin S: n \sim m \text { for some } m \in S\} .
$$

We obtain estimates on the spectrum of $H$ which depend mainly upon the number $k:=$ $\#(\partial S)$. One has $k=2$ if $S$ is an interval in $\mathbf{Z}$, the only case considered by Arveson, but in higher dimensions $k$ increases indefinitely as $S$ expands. One immediately sees that $\operatorname{rank}\{(1-P) H P\} \leqslant k$. We use this fact to compare the spectrum of $H$ with that of its restriction $B:=P H P$ to $\mathcal{L}$.

The following theorem is applicable to the example above, but we have formulated it in a more general manner.

Theorem 10. If $\operatorname{rank}\{(1-P) H P\} \leqslant k$ then

$$
F(s) \leqslant d_{k+1, B}(s)
$$

for all $s \in \mathbf{R}$. Hence

$$
\operatorname{Spec}(H) \cap\left[\lambda_{i}, \lambda_{i+k}\right] \neq \emptyset
$$

for all $i$, where $\left\{\lambda_{i}\right\}$ are the eigenvalues of $B$ written in increasing order and repeated according to multiplicity.

Proof. If $\left\{\mu_{i}\right\}$ are the eigenvalues of $(B-s 1)^{2}$, written in increasing order and repeated according to multiplicity, then

$$
\begin{aligned}
d_{k+1, B}(s)^{2} & =\mu_{k+1}(s) \\
& =\inf \left\{\mu(L): L \in \varepsilon_{k+1}\right\}
\end{aligned}
$$


where $\mathcal{E}_{k+1}$ is the set of linear subspaces of $\mathcal{L}$ of dimension $k+1$ and

$$
\mu(L):=\sup \left\{\frac{\|B f-s f\|^{2}}{\|f\|^{2}}: 0 \neq f \in L\right\} .
$$

If $\operatorname{dim}(L)=k+1$, then there exists $f \in L$ with $\|f\|=1$ and $f \in \operatorname{Ker}\{(1-P) H P\}$. We deduce that

$$
F(s)^{2} \leqslant\langle N(s) f, f\rangle=\|B f-s f\|^{2} \leqslant \mu(L) .
$$

Taking the inf over all $L \in \mathcal{E}_{k+1}$ yields the first statement of the theorem. The second statement is obtained by putting $s:=\left(\lambda_{i}+\lambda_{i+k}\right) / 2$ to obtain $d_{k+1, B}(s)=\left(\lambda_{i+k}-\lambda_{i}\right) / 2$. From the inequality

$$
F\left(\frac{\lambda_{i}+\lambda_{i+k}}{2}\right) \leqslant \frac{\lambda_{i+k}-\lambda_{i}}{2}
$$

we complete the proof by invoking Corollary 2 .

The same idea can be applied using the functions $F_{n}(s)$ to obtain the following result, but we write the proof in a more direct form.

Theorem 11. Let $-\infty<\alpha<\beta<\infty$ and suppose that $B$ has $j$ eigenvalues in $[\alpha, \beta]$. Then either there is a point of the essential spectrum of $H$ in $[\alpha, \beta]$ or $H$ has at least $j-k$ eigenvalues in $[\alpha, \beta]$.

Proof. If $\mathcal{L} \subseteq \mathcal{L}$ is the linear span of the eigenvectors of $B$ associated with the eigenvalues lying in $[\alpha, \beta]$ then $\operatorname{dim}(\mathcal{L})=j$. If we put $\gamma:=(\alpha+\beta) / 2$ and $\delta:=(\beta-\alpha) / 2$, then $\|B f-\gamma f\| \leqslant \delta\|f\|$ for all $f \in \mathcal{L}$. If $\mathcal{M}$ is the subspace consisting of all $f \in \mathcal{L}$ such that $(1-P) H P f=0$ then $\operatorname{dim}(\mathcal{M}) \geqslant j-k$ and $H f=B f$ for all $f \in \mathcal{M}$. Thus $\|H f-\gamma f\| \leqslant \delta\|f\|$ for all $f \in \mathcal{M}$. Applying the Rayleigh-Ritz principle to the operator $|H-\gamma 1|$ we deduce that $H$ has at least $j-k$ eigenvalues (or non-empty essential spectrum) in the stated interval.

We interpret the above theorem by saying that in the passage from $B$ to $H$ one loses at most $k$ eigenvalues in each interval, regardless of the length of that interval. However, the remaining eigevalues may also move within the interval, and new eigenvalues, or even essential spectrum of $H$, may appear.

As an example, let $H$ be the operator on $l^{2}\left(\mathbf{Z}^{N}\right)$ defined above, so that $\|H\| \leqslant c$. Let $S:=[-m, m]^{N} \cap \mathbf{Z}^{N}$ so that $\# S=(2 m+1)^{N}$ and $k:=\#(\partial S) \leqslant 2 N(2 m+2)^{N-1}$. If we divide $[-c, c]$ into a fixed number $h$ of equal subintervals, then we might have up to $k$ spurious eigenvalues in each subinterval, and hence might have up to $h k$ spurious eigenvalues altogether. The proportion of spurious eigenvalues lost is therefore at most $O\left(m^{-1}\right)$.

The computation of the eigenvalues of $B:=P H P$ can be carried out particularly efficiently in one dimension, since $B$ is then a tri-diagonal matrix. Numerical examples can be extracted from Arveson's program for computing the spectrum of the almost Mathieu operator [3]. This operator on $l^{2}(\mathbf{Z})$ has matrix elements

$$
H_{m, n}:=\left\{\begin{array}{cl}
1 & \text { if }|m-n|=1 \\
\lambda \cos (n \theta) & \text { if } m=n \\
0 & \text { otherwise. }
\end{array}\right.
$$


It has only essential spectrum, but a more precise classification depends delicately upon $\lambda$ and $\theta$, the interesting cases being when $\theta$ is irrational $\bmod 2 \pi$. Arveson's program provides beautiful evidence of the extremely complicated spectral behaviour of this operator. If a particular example is saved, then the data file consists of a list of the number of eigenvalues in each of a substantial number of sub-intervals of the real line. The data is stored as an unadorned hexadecimal list, but this is easy to interpret. We refer to [6], [24], [25] for references to the extensive theoretical literature on the almost Mathieu equation.

For the reader's convenience we have recreated the mathematical core of Arveson's program using Maple V.4 [10]. His key idea is to compute the number of eigenvalues of $B$ less than $s$ for enough values of $s$ to produce a graph (see Figure 3). This may be done very efficiently using the slicing method [31, p. 371]. Differences of successive elements of the data list yield the number of eigenvalues of $B$ in each of a series of consecutive intervals. The efficiency of the method arises from the fact that there is no need to take the number of such intervals to be larger than the number of horizontal pixels of the graph to be drawn, however large the dimension of the subspace.

\section{Schrödinger operators}

Let $H:=-\Delta+V$ be a Schrödinger operator acting in $L^{2}\left(\mathbf{R}^{N}\right)$, and let its potential $V$ be non-negative and locally $L^{2}$, so that $H$ is essentially self-adjoint on $C_{c}^{\infty}\left(\mathbf{R}^{N}\right)$. The computation of the spectrum of $H$ is difficult because it depends upon the entire structure of the potential $V$. The theorems which we obtain are continuous analogues of Proposition 7.1 of Avron, van Mouche and Simon [6], which was used by them to prove that the spectrum of an almost Mathieu equation is a Holder continuous function of the phase parameter. This may then be used to compute the spectrum by approximating the phase by a rational number, for which a detailed theory has been developed; see [24], [25] for the best recent results and a review of the literature.

We start by proving a theorem about the bottom of the spectrum of $H$. While this is a special case of the subsequent result, its proof is simpler and the constant obtained is more precise. The theorem is surprising in view of the fact that the bottom of the spectrum of $H$ may have an associated eigenfunction which is concentrated on a region of arbitrarily large diameter. It is related to Theorem 2 of [15], which computes the weak coupling asymptotics of the bottom of the spectrum for arbitrary bounded potentials.

Let $E$ denote the bottom of the spectrum of $H$, and $E_{x, L}$ the bottom of the spectrum of the restriction of $H$ to $L^{2}(B(x, L))$ subject to Dirichlet boundary conditions, where $B(x, L)$ denotes the ball in $\mathbf{R}^{N}$ with centre $x$ and radius $L$. Finally, let

$$
E_{L}:=\inf \left\{E_{x, L}: x \in \mathbf{R}^{N}\right\} .
$$

Theorem 12. Under the above assumptions we have

$$
E_{L}^{1 / 2}-L^{-1} \gamma^{1 / 2} \leqslant E^{1 / 2} \leqslant E_{L}^{1 / 2}
$$

for all $L>0$, where $\gamma$ is the smallest eigenvalue of $-\Delta$ acting in $L^{2}(B(0,1))$ subject to Dirichlet boundary conditions.

Proof. Let $\eta$ be a smooth function on $\mathbf{R}^{N}$ with support in $B(0,1)$ and $\|\eta\|_{2}=1$. Let

$$
\eta_{x, L}(y):=L^{-N / 2} \eta\left(L^{-1}(y-x)\right)
$$




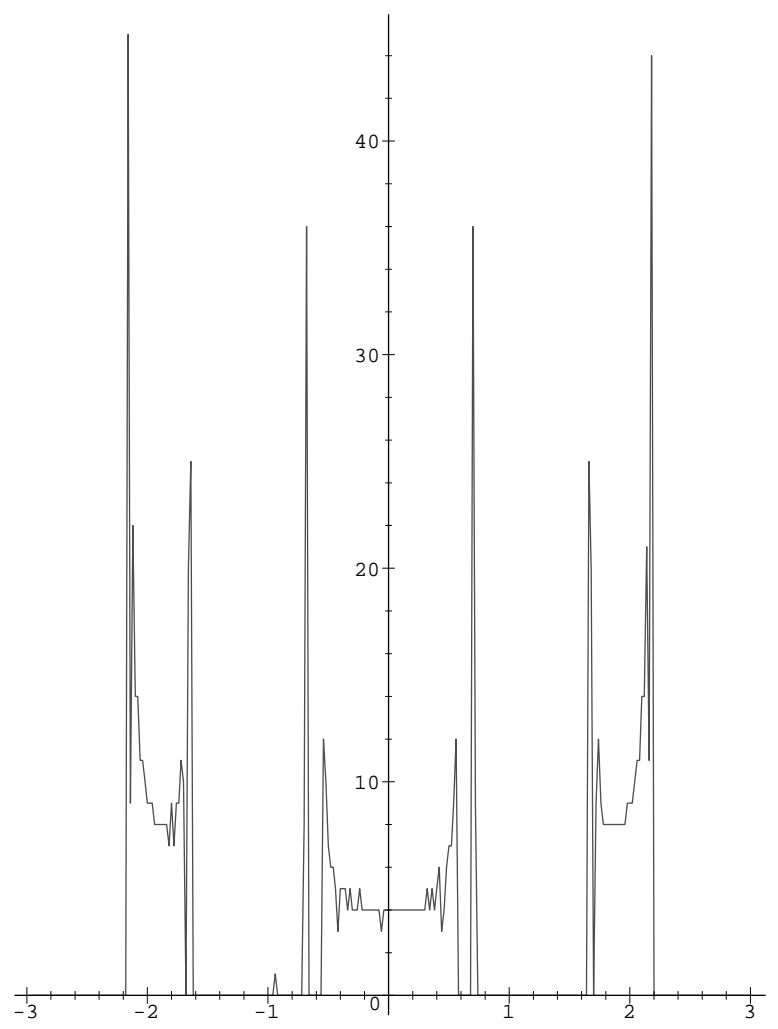

Figure 3: Spectrum of an almost Mathieu operator 
and regard $\eta_{x, L}$ as a multiplication operator. A direct computation establishes that

$$
\int_{\mathbf{R}^{N}}\left(\eta_{x, L}\right)^{*}\left(\eta_{x, L}\right) \mathrm{d} x=\|\eta\|_{2}^{2} 1=1
$$

where the integral converges in the weak operator topology. Let $Q$ be the quadratic form of $H$ with domain

$$
\operatorname{Dom}(Q)=W^{1,2}\left(\mathbf{R}^{N}\right) \cap \operatorname{Dom}\left(V^{1 / 2}\right)
$$

and form core $C_{c}^{\infty}$. Let $\varepsilon, \delta>0$ and let $\phi \in C_{c}^{\infty}$ satisfy

$$
Q(\phi) \leqslant(E+\varepsilon)\|\phi\|^{2} .
$$

Then

$$
\begin{aligned}
\int_{\mathbf{R}^{N}} Q\left(\eta_{x, L} \phi\right) \mathrm{d} x= & \int_{\mathbf{R}^{N}}\left(\left\|\nabla\left(\eta_{x, L} \phi\right)\right\|^{2}+\left\|V^{1 / 2} \eta_{x, L} \phi\right\|^{2}\right) \mathrm{d} x \\
= & \int_{\mathbf{R}^{N}}\left(\left\|\nabla\left(\eta_{x, L}\right) \phi+\eta_{x, L} \nabla(\phi)\right\|^{2}+\left\|V^{1 / 2} \eta_{x, L} \phi\right\|^{2}\right) \mathrm{d} x \\
\leqslant & \left(1+\delta^{-1}\right) \int_{\mathbf{R}^{N}}\left\|\nabla\left(\eta_{x, L}\right) \phi\right\|^{2} \mathrm{~d} x+(1+\delta) \int_{\mathbf{R}^{N}}\left\|\eta_{x, L} \nabla(\phi)\right\|^{2} \mathrm{~d} x \\
& +\int_{\mathbf{R}^{N}}\left\|V^{1 / 2} \eta_{x, L} \phi\right\|^{2} \mathrm{~d} x \\
\leqslant & \left(1+\delta^{-1}\right) L^{-2}\|\nabla \eta\|^{2}\|\phi\|^{2}+(1+\delta) Q(\phi) \\
\leqslant & \left(\left(1+\delta^{-1}\right) L^{-2}\|\nabla \eta\|^{2}+(1+\delta)(E+\varepsilon)\right)\|\phi\|^{2} \\
= & \left(\left(1+\delta^{-1}\right) L^{-2}\|\nabla \eta\|^{2}+(1+\delta)(E+\varepsilon)\right) \int_{\mathbf{R}^{N}}\left\|\eta_{x, L} \phi\right\|^{2} \mathrm{~d} x .
\end{aligned}
$$

The above inequality implies that there exists $x \in \mathbf{R}^{N}$ such that

$$
E_{x, L} \leqslant\left(1+\delta^{-1}\right) L^{-2}\|\nabla \eta\|^{2}+(1+\delta)(E+\varepsilon)
$$

and hence that

$$
E_{L} \leqslant\left(1+\delta^{-1}\right) L^{-2}\|\nabla \eta\|^{2}+(1+\delta)(E+\varepsilon) .
$$

By letting $\varepsilon \rightarrow 0$ and then optimising with respect to $\delta$, we obtain

$$
E_{L}^{1 / 2} \leqslant L^{-1}\|\nabla \eta\|+E^{1 / 2}
$$

The theorem follows by optimising with respect to $\eta$.

We now turn to the computation of the entire spectrum of $H$. Let

$$
F(s):=\operatorname{dist}(s, \operatorname{Spec}(H))
$$

and put

$$
F_{x, L}(s):=\inf \left\{\frac{\|H \phi-s \phi\|}{\|\phi\|}: 0 \neq \phi \in \mathcal{D}_{x, L}\right\} .
$$

where $\mathscr{D}_{x, L}$ denotes the space of smooth functions with compact support in $B(x, L)$. We adopt the position that $F_{x, L}$ may be computed by means which depend upon the operator in question, and describe how these functions may be used to obtain estimates of $\operatorname{Spec}(H)$ with controlled errors. 
Although it is fairly obvious that $F_{0, L}(s)$ decreases monotonically and locally uniformly to $F(s)$ as $L \rightarrow \infty$, the rate of convergence cannot be controlled without assumptions on $V$. If we put

$$
F_{L}(s):=\inf _{x \in \mathbf{R}^{N}}\left\{F_{x, L}(s)\right\}
$$

then this function also decreases monotonically and locally uniformly to $F(s)$. However, the rate of convergence can now be controlled, without any further assumptions on $V$.

Theorem 13. There exists $c>0$ depending only upon $N$ such that

$$
F(s) \leqslant F_{L}(s) \leqslant F(s)+c\left(L^{-2}+L^{-1}(s+F(s))^{1 / 2}\right)
$$

for all $s \in \mathbf{R}$. Hence

$$
F_{L}(s)-c\left(L^{-2}+L^{-1}\left(s+F_{L}(s)\right)^{1 / 2}\right) \leqslant F(s) \leqslant F_{L}(s)
$$

Proof. We follow the notation of Theorem 12. Let $\delta, \varepsilon>0$ and assume that $\phi \in C_{c}^{\infty}$ satisfies $\|(H-s) \phi\| \leqslant(F(s)+\varepsilon)\|\phi\|$. Then

$$
\begin{aligned}
\int_{\mathbf{R}^{N}}\left\|(H-s) \eta_{x, L} \phi\right\|^{2} \mathrm{~d} x \leqslant & \left(1+\delta^{-1}\right) \int_{\mathbf{R}^{N}}\left\|\eta_{x, L}(H-s) \phi\right\|^{2} \mathrm{~d} x \\
& +(1+\delta) \int_{\mathbf{R}^{N}}\left\|\left[H, \eta_{x, L}\right] \phi\right\|^{2} \mathrm{~d} x .
\end{aligned}
$$

Secondly if $\gamma>0$

$$
\begin{aligned}
\int_{\mathbf{R}^{N}}\left\|\left[H, \eta_{x, L}\right] \phi\right\|^{2} \mathrm{~d} x= & \int_{\mathbf{R}^{N}}\left\|\left(\Delta \eta_{x, L}\right) \phi+2 \nabla \eta_{x, L} \cdot \nabla \phi\right\|^{2} \mathrm{~d} x \\
\leqslant & \left(1+\gamma^{-1}\right) \int_{\mathbf{R}^{N}}\left\|\left(\Delta \eta_{x, L}\right) \phi\right\|^{2} \mathrm{~d} x \\
& +4(1+\gamma) \int_{\mathbf{R}^{N}}\left\|\left(\nabla \eta_{x, L}\right) \cdot \nabla \phi\right\|^{2} \mathrm{~d} x \\
\leqslant & c_{1}\left(1+\gamma^{-1}\right) L^{-4}\|\phi\|^{2}+c_{2}(1+\gamma) L^{-2}\|\nabla \phi\|^{2} \\
\leqslant & \left(c_{1}\left(1+\gamma^{-1}\right) L^{-4}+c_{2}(1+\gamma) L^{-2}(s+F(s)+\varepsilon)\right)\|\phi\|^{2} \\
= & c_{3}^{2}\left(L^{-2}+L^{-1}(s+F(s)+\varepsilon)^{1 / 2}\right)^{2}\|\phi\|^{2}
\end{aligned}
$$

by optimising with respect to $\gamma$. Combining the above two inequalities and optimising with 
respect to $\delta$ we obtain

$$
\begin{aligned}
\int_{\mathbf{R}^{N}}\left\|(H-s) \eta_{x, L} \phi\right\|^{2} \mathrm{~d} x \leqslant & \left(1+\delta^{-1}\right)\|(H-s) \phi\|^{2} \\
& +(1+\delta) c_{3}^{2}\left(L^{-2}+L^{-1}(s+F(s)+\varepsilon)^{1 / 2}\right)^{2}\|\phi\|^{2} \\
\leqslant & \left(1+\delta^{-1}\right)(F(s)+\varepsilon)^{2}\|\phi\|^{2} \\
& +(1+\delta) c_{3}^{2}\left(L^{-2}+L^{-1}(s+F(s)+\varepsilon)^{1 / 2}\right)^{2}\|\phi\|^{2} \\
= & k^{2}\|\phi\|^{2} \\
= & k^{2} \int_{\mathbf{R}^{N}}\left\|\eta_{x, L} \phi\right\|^{2} \mathrm{~d} x
\end{aligned}
$$

where

$$
k:=(F(s)+\varepsilon)+c_{3}\left(L^{-2}+L^{-1}(s+F(s)+\varepsilon)^{1 / 2}\right) .
$$

This integral inequality implies the existence of $x \in \mathbf{R}^{N}$ such that

$$
\left\|(H-s) \eta_{x, L} \phi\right\| \leqslant k\left\|\eta_{x, L} \phi\right\| .
$$

The proof is completed by taking the imfimum over $x \in \mathbf{R}^{N}$ and then the limit $\varepsilon \rightarrow 0$.

Our next result is a partial converse to Corollary 2.

Corollary 14. If $E \in \operatorname{Spec}(H)$ and $L>0$, then there exist $x \in \mathbf{R}^{N}$ and $\phi \in \mathcal{D}_{x, L}$ such that

$$
\frac{\|H \phi-E \phi\|}{\|\phi\|} \leqslant c\left(L^{-2}+L^{-1} E^{1 / 2}\right) .
$$

Proof. The stated condition on $E$ implies that $F(E)=0$, which implies the result immediately.

If one has further information about the potential $V$ then it may be preferable to use a partition of the identity which takes advantage of this. For example suppose that $V$ is periodic except for sparsely distributed compactly supported impurities. Then one could take a different function in the partition of the identity to equal one on the support of each impurity. If every pair of impurities is separated by a distance of at least $O(L)$, then we may take $\varepsilon_{1}=O\left(L^{-2}\right)$ and $\varepsilon_{2}=O\left(L^{-4}\right)$ below.

Theorem 15. Suppose $\left\{\eta_{n}\right\}_{n=1}^{\infty}$ lie in $C_{c}^{\infty}\left(\mathbf{R}^{N}\right)$ with

$$
\begin{gathered}
\sum_{n=1}^{\infty} \eta_{n}(x)^{2}=1 \\
\sum_{n=1}^{\infty}\left|\nabla \eta_{n}(x)\right|^{2} \leqslant \varepsilon_{1} \\
\sum_{n=1}^{\infty}\left|\Delta \eta_{n}(x)\right|^{2} \leqslant \varepsilon_{2}
\end{gathered}
$$


for all $x \in \mathbf{R}^{N}$. Define

$$
F_{n}(s):=\inf \left\{\frac{\|H \phi-s \phi\|}{\|\phi\|}: 0 \neq \phi_{n} \in C_{c}^{\infty}\left(U_{n}\right)\right\}
$$

where $U_{n}$ is some open set containing $\operatorname{supp}\left\{\eta_{n}\right\}$, and

$$
\bar{F}(s):=\inf \left\{F_{n}(s): n=1,2, . .\right\} .
$$

Then

$$
\bar{F}(s)-\varepsilon_{2}-2 \varepsilon_{1}\{s+\bar{F}(s)\}^{1 / 2} \leqslant F(s) \leqslant \bar{F}(s)
$$

for all $s \in \mathbf{R}$.

We omit the proof, which follows that of Theorem 13.

\section{Complex resonances}

The standard theory of complex resonances is easier to use than it is to explain in fundamental terms. It involves the notion that certain self-adjoint operators may have complex eigenvalues associated with eigenvectors which do not lie in the Hilbert space under consideration. The problem with this idea is that one must then impose constraints on the eigenvectors involved, in order to prevent every complex number being a resonance, and these constraints can appear rather arbitrary.

One extensively developed theory of resonances is described in the review by [8] of ideas first developed by Aguilar, Balslev, Combes and Simon in the early 1970s. The authors use a dense linear subspace to define resonances of a Schrödinger operator, namely the subspace of analytic vectors with respect to the dilation group acting on $\mathbf{R}^{N}$; see $[8, \mathrm{p}$. 152]. Resonances are defined as poles of the analytic continuation of the resolvent to the unphysical sheet. This definition is canonical to the extent that one does not distinguish between the resolvent operator and either its integral kernel or its matrix elements with respect to the linear subspace. While technically successful for certain types of Schrödinger operator, it cannot be used if the configuration space is a manifold or a discrete set, because of the absence of a dilation group in these cases. A more general definition applicable to Laplace-Beltrami operators on Riemannian manifolds has been proposed by Agmon [2], [1], and again depends upon the choice of a dense linear subspace satisfying a certain list of properties. Both definitions have the feature that the theory depends upon the spectrum of $H$ having an interval of absolute continuity, and they are therefore unstable with respect to arbitrarily small perturbations. For example, if one adds a very small almost periodic perturbation to a Schrödinger operator, the above notion of resonance becomes meaningless.

In the next section we develop a different notion of resonance, in which the role of the subspace is absolutely central, and in which analytic continuation of the resolvent plays no role. Both approaches are applicable in a multi-dimensional context, but we investigate some one-dimensional examples here in order to compare the two.

We consider a discrete Schrödinger operator $H$ acting on $l^{2}\left(\mathbf{Z}^{+}\right)$. This operator is bounded and has matrix

$$
H_{m, n}:=\left\{\begin{array}{cl}
1 & \text { if }|m-n|=1 \\
v_{n} & \text { if } m=n \\
0 & \text { otherwise }
\end{array}\right.
$$


where the potential $\left\{v_{n}\right\}_{n=1}^{\infty}$ is real-valued and of finite support. We assume that $v_{k} \neq 0$ but that $v_{n}=0$ for all $n>k$. An application of trace class scattering theory shows that the operator $H$ has essential spectrum and absolutely continuous spectrum equal to [-2, 2].

Adapting the definition of resonance of Harrell [18] to this discrete context, we say that $z \in \mathbf{C}$ is a resonance of $H$ if the solution $f$ of $H f=z f$ subject to the intial condition $f_{1}=1$ satisfies $f_{n}=c w^{n}$ for some $c, w \in \mathbf{C}$ and all large enough $n$. We do not impose the condition $f \in l^{2}$ but merely regard $f$ as a sequence $\left\{f_{n}\right\}_{n=1}^{\infty}$.

Theorem 16. If $z$ is a complex resonance, then $z=w^{-1}+w$ and the condition $f_{n}=c w^{n}$ holds for all $n \geqslant k$. Moreover, $z$ is a solution of a polynomial equation $p(z)=0$ of degree $2 k-1$.

Proof. From the equations

$$
f_{n-1}+v_{n} f_{n}+f_{n+1}=z f_{n}
$$

where we put $f_{0}=0$ and $f_{1}=1$, we deduce that $f_{n}$ is a polynomial of the form

$$
f_{n}=z^{n-1}-\left(v_{1}+\ldots+v_{n-1}\right) z^{n-2}+O\left(z^{n-3}\right) .
$$

If $n \geqslant k$ then

$$
f_{n}+f_{n+2}=z f_{n+1}
$$

and inserting the expression for $f_{n}$ for large $n$ we deduce that $z=w^{-1}+w$. Carrying out a reverse induction from such large $n$, we find that $f_{n}=c w^{n}$ for all $n \geqslant k$.

We now combine the two equations $z=w^{-1}+w$ and $f_{k+1} / f_{k}=w$ to obtain

$$
p(z):=z f_{k+1} f_{k}-f_{k}^{2}-f_{k+1}^{2}=0 .
$$

Combining (8.1) and (8.2) we finally obtain

$$
p(z)=v_{k} z^{2 k-1}+O\left(z^{2 k-2}\right)
$$

which confirms the order of $p$.

It is generically the case that the roots of a polynomial depend analytically upon the coefficients, and in our context this means that they depend analytically upon the coefficients of the potential. However, there is an important exception. If one examines the dependence of the roots upon the value of $v_{k}$, one sees that when $v_{k}=0$ the degree of the polynomial decreases, so some of the roots momentarily disappear. In fact, as $v_{k} \rightarrow 0$ certain of the resonances become rapidly larger, moving to infinity.

In the program [11] we investigate the case in which $v_{1}=0, v_{2}=3$ and $v_{3}=a$ varies through the value zero, while all other $v_{n}=0$. Thus $k=3$ and there are 5 resonances, except when $a=0$, when there are only 3 resonances. For $a=0$ the polynomial equation mentioned in Theorem 16 is

$$
p(z)=3 z^{3}-9 z^{2}-6 z-1
$$

Table 3 shows how two selected resonances depend upon $a$ when this is very small. More complete data can be obtained from [11], for this and other potentials. Moreover [11] provides a procedure for plotting the data obtained in the complex plane. It may be seen that one of the resonances diverges as $a \rightarrow 0$, while the other varies smoothly under the same conditions. This is confirmed by further numerical experiments. 
Table 3:

\begin{tabular}{ccc}
\hline$a$ & resonance 1 & resonance 2 \\
\hline-.08 & $-.28475+.085342 i$ & 7.9289 \\
-.07 & $-.28562+.085732 i$ & 8.3343 \\
-.06 & $-.28649+.086119 i$ & 8.8411 \\
-.05 & $-.28738+.086479 i$ & 9.4942 \\
-.04 & $-.28827+.086827 i$ & 10.385 \\
-.03 & $-.28919+.087146 i$ & 11.696 \\
-.02 & $-.29012+.087453 i$ & 13.909 \\
-.01 & $-.29105+.087747 i$ & 18.939 \\
0 & $-.29199+.088010 i$ & $\infty$ \\
.01 & $-.29294+.088259 i$ & $1.5058+17.199 i$ \\
.02 & $-.29392+.088476 i$ & $1.5109+12.074 i$ \\
.03 & $-.29489+.088679 i$ & $1.5165+9.7876 i$ \\
.04 & $-.29586+.088867 i$ & $1.5225+8.4166 i$ \\
.05 & $-.29684+.089021 i$ & $1.5279+7.4733 i$ \\
.06 & $-.29783+.089160 i$ & $1.5336+6.7732 i$ \\
.07 & $-.29883+.089264 i$ & $1.5388+6.2243 i$ \\
.08 & $-.29983+.089353 i$ & $1.5444+5.7798 i$ \\
\hline
\end{tabular}

\section{Higher-order spectra}

Let $H$ be a self-adjoint operator acting on a Hilbert space $\mathscr{H}$, and let $P$ be an orthogonal projection onto a closed linear subspace $\mathcal{L}$. We assume either that $H$ is bounded, or that $\mathcal{L}$ is finite-dimensional and contained in the domain of as high a power of $H$ as is needed for each statement. Given a positive integer $n$ and $z \in \mathbf{C}$, let $M_{n}(z)$ denote the restriction to $\mathcal{L}$ of the operator pencil $P(H-z I)^{n} P$. We define the $n$-th order $\operatorname{spectrum} \operatorname{Spec}_{n}(H, \mathcal{L})$ of $H$ relative to $\mathcal{L}$ to be the set of $z$ such that the operator $M_{n}(z)$ is not invertible within the subspace $\mathcal{L}$.

Theorem 17. The set $\operatorname{Spec}_{n}(H, \mathcal{L})$ is non-empty, closed and bounded for all positive integers $n$.

Proof. If $\mathcal{L}$ is finite-dimensional this is an immediate consequence of the fact that $z \in$ $\operatorname{Spec}_{n}(H, \mathcal{L})$ if and only if the characteristic polynomial

$$
p_{n}(z):=\operatorname{det}\left(M_{n}(z)\right)
$$

vanishes. Note that $p_{n}$ is of degree $n \operatorname{dim}(\mathscr{L})$.

Now suppose that $H$ is bounded, but that $\mathcal{L}$ is infinite-dimensional. Then we may follow the same argument for $n>1$ as in the traditional case where $n=1$ and $\mathcal{L}=\mathscr{H}$. The boundedness of the spectrum is a consequence of the estimate

$$
\left\|(-z)^{-n} M_{n}(z)-I\right\| \leqslant \sum_{r=1}^{n}\left(\begin{array}{l}
n \\
r
\end{array}\right) \frac{\|H\|^{r}}{|z|^{r}} .
$$


Its closedness follows from the fact that $M_{n}(z)$ depends norm continuously on $z$. The proof of its non-emptiness involves an application of Liouville's theorem to $M_{n}(z)^{-1}$, assuming that this inverse is everywhere defined.

Our next theorem is not sharp. It would be interesting to know the strongest restriction which may be placed on $\operatorname{Spec}_{2}(H, \mathcal{L})$ under the given assumption on $\operatorname{Spec}(H)$.

Theorem 18. Suppose that $H$ is bounded with $\operatorname{Spec}(H) \subseteq[\alpha, \beta]$. Then

$$
\operatorname{Spec}_{2}(H, \mathcal{L}) \subseteq\{z \in \mathbf{C}:|z| \leqslant 3\|H\| \text { and } \alpha \leqslant \operatorname{Re}(z) \leqslant \beta\} .
$$

Proof. The first part of the statement follows from the estimate (9.2).

Now suppose that $z=x+i y$ where $x<\alpha$, and let $\phi \in \mathcal{L}$. Then

$$
\begin{aligned}
\left\|M_{2}(z) \phi\right\|\|\phi\| & \geqslant\left|\left\langle M_{2}(z) \phi, \phi\right\rangle\right| \\
& =\left|\left\langle\left[(H-x)^{2}-y^{2}-2 i y(H-x)\right] \phi, \phi\right\rangle\right| \\
& \geqslant 2|y|\langle(H-x) \phi, \phi\rangle \mid \\
& \geqslant 2|y|(\alpha-x)\|\phi\|^{2} .
\end{aligned}
$$

If $y \neq 0$, then we deduce that there exists $c>0$ such that

$$
\left\|M_{2}(z) \phi\right\| \geqslant c\|\phi\|
$$

for all $\phi \in \mathcal{L}$, so that $M_{2}(z)$ is one-one with closed range. If $\psi$ is orthogonal to the range, then

$$
0=\left|\left\langle M_{2}(z) \psi, \psi\right\rangle\right| \geqslant 2|y|(\alpha-x)\|\psi\|^{2} .
$$

Therefore $\psi=0, M_{2}(z)$ is invertible and $z \notin \operatorname{Spec}_{2}(H, \mathcal{L})$. If $y=0$, then an easier version of the above calculation again shows that $z \notin \operatorname{Spec}_{2}(H, \mathcal{L})$. A similar argument works if $x>\beta$.

We define the resonances of $H$ relative to $\mathcal{L}$ to be those isolated points $z \in \operatorname{Spec}_{2}(H, \mathcal{L})$ such that 0 is an isolated eigenvalue of $M_{2}(z)$ of finite multiplicity; we are particularly interested in those points which are close to the real axis. If $\operatorname{dim}(\mathcal{L})<\infty$ then every point of $\operatorname{Spec}_{2}(H, \mathcal{L})$ is a resonance. Note that an eigenvalue of $H$ may lead to a resonance in this sense, because the associated eigenfunction may be close to $\mathcal{L}$ but not an element of $\mathcal{L}$. There is no way of avoiding this fact-the use of the subspace $\mathcal{L}$ degrades the information contained in $H$. Alternatively two different self-adjoint extensions of $\left.H\right|_{\mathscr{L}}$ may have different spectral characteristics, and we are investigating only what is common to all possible such extensions.

The next theorem shows that our new definition of resonance is stable under small perturbations.

Theorem 19. Let $a \in \mathbf{C}$ be a resonance of $H$ with respect to $\mathcal{L}$. Then for every $\varepsilon>0$ there exists $\delta>0$ such that if $A: \mathscr{H} \rightarrow \mathcal{H}$ is bounded and self-adjoint with $\|A\|<\delta$, then there exists a resonance eigenvalue $z$ of $H+A$ such that $|z-a|<\varepsilon$.

Proof. If $\operatorname{dim}(\mathscr{L})<\infty$ then we need only apply Rouché's theorem to the polynomial $p_{2}$ defined by (9.1) since the coefficients of $p_{2}$ vary continuously with the perturbation. If $\operatorname{dim}(\mathcal{L})=\infty$ and $H$ is bounded, we must make substantial use of Kato's theory of 
holomorphic perturbations of the spectrum [20]. The spectral projection $P_{a}$ associated with the resonance $a$ is given by the contour integral

$$
P_{a}:=\frac{1}{2 \pi i} \int_{\gamma}\left(M_{2}(a)-w\right)^{-1} \mathrm{~d} w
$$

where $\gamma$ is a sufficiently small circle with centre 0 . This projection is of finite rank and commutes with $M_{2}(a)$. If we fix $\gamma$ then for $|z-a|$ sufficiently small the projections

$$
P_{z}:=\frac{1}{2 \pi i} \int_{\gamma}\left(M_{2}(z)-w\right)^{-1} \mathrm{~d} w
$$

depend analytically upon $z$, and therefore have the same rank as for $z=a$. The function

$$
G(z):=\operatorname{det}\left(M_{2}(z) P(z)\right)
$$

is defined by computing the determinant within the range of $P(z)$, and again depends analytically upon $z$. Since $a$ is a resonance, this function vanishes for $z=a$ but is non-zero everywhere else in a sufficiently small neighbourhood of $a$. Now let us consider the same constructions for $\tilde{H}:=H+A$. If $\|A\|$ is small enough, then $\tilde{G}(z)$ can be defined as before in a small neighbourhood of $a$, and is uniformly close to $G(z)$. By Rouché's theorem it has an isolated zero in a neighbourhood of $a$, and this yields a resonance of $\tilde{H}$.

We now turn to connecting this account of resonance to the theory of Section 2, using the notation developed there. If $s \in \mathbf{R}$ then Theorem 5 states that

$$
F(s)^{-2}=\left\|M_{2}(s)^{-1}\right\| \text {. }
$$

One might expect $F(s)$ to be small close to a point $z \in \mathbf{C}$ at which $M_{2}(z)$ is noninvertible, i.e. close to a resonance of $H$. If $\mathcal{L}$ is finite-dimensional, this can be made more precise. Instead of asking whether the smallest eigenvalue $F(s)$ of $M_{2}(s)$ is small, one may ask whether its determinant is small. But this determinant is the polynomial of degree $n \operatorname{dim}(\mathcal{L})$ whose extension to $\mathbf{C}$ vanishes at the resonances of $H$ relative to $\mathcal{L}$.

There is more which needs to be understood about the above idea, at both theoretical and computational levels, but we consider next an example which demonstrates that the theory above really is related to complex resonances in the sense of Section 8.

Example 20. Let $H$ be the bounded self-adjoint operator acting in $l^{2}\left(\mathbf{Z}^{+}\right)$, whose matrix is given by

$$
H_{m, n}:= \begin{cases}1 & \text { if }|m-n|=1 \\ a & \text { if } m=n=3 \\ 0 & \text { otherwise }\end{cases}
$$

for $m, n \geqslant 1$ and $a>0$. This operator has absolutely continuous spectrum [-2,2] with multiplicity 1 , together with a single eigenvalue $\lambda>2$. If we define resonances as in Section 8 , then they are the roots of the polynomial equation

$$
p(z):=-a z^{5}+a^{2} z^{4}+4 a z^{3}-2 a^{2} z^{2}-3 a z+a^{2}+1=0
$$

of degree 5 in $z$. If $a=3$, the roots are approximately

$$
\begin{array}{r}
-1.1421 \pm 0.0342 i \\
0.8403 \pm 0.0489 i
\end{array}
$$

3.6036 . 
The first four roots are complex resonances, and the last is the $l^{2}$ eigenvalue $\lambda$.

Now let us describe the approach of this section. Given any integer $k>1$, one can define $\mathcal{L}$ to be the subspace of functions with support in $\{1, \ldots, k\}$. For reasonably small $k$ one can compute $p_{2}(z)$ as defined by (9.1) and find the zeros of the polynomial $p_{2}$ by the use of a computer algebra package. (The only merit of this method is the simplicity of the programming involved. It is numerically unstable for large values of $k$ because the coefficients of $p_{2}$ become so large. We suggest four alternative methods of computing the complex resonances below.)

We have investigated the above example numerically for $a=3$ and $k=20$ using the method just described and also the method of Theorem 23 below [12]. The graph of $F(s)$ for real $s$ in Figure 4 shows clear local minima near -1.14 and 0.84 , as well as an eigenvalue near 3.60, that is at the resonances as well as at the $l^{2}$ eigenvalue. However, the first two local minima mentioned are not of great value for locating the resonances in the complex plane. The graph plotting the complex resonances as defined in this paper, shown in Figure 5 is extremely interesting.

Most of the points lie near a closed convex curve, corresponding in some way to the continuous spectrum of $H$. There is a pair of points at $3.6036 \pm 10^{-8} i$ very close to the $l^{2}$ eigenvalue of $H$, which is of multiplicity 1 . Finally there are four complex resonances at

$$
\begin{array}{r}
-1.1425 \pm 0.0600 i \\
0.8357 \pm 0.0793 i
\end{array}
$$

It is rather surprising that these four complex resonances vary only very slowly with $k$, in contrast to the other points of the second-order spectrum of $H$. For example, if we put $k=4$ the corresponding values are

$$
\begin{array}{r}
-1.1527 \pm 0.1306 i \\
0.8500 \pm 0.1707 i .
\end{array}
$$

We do not claim that our approach is superior to the standard one for this example, but emphasise again that our result applies equally to all self-adjoint extensions of $\left.H\right|_{\mathcal{L}}$. This elementary procedure, which could still be implemented if one added a small almost periodic potential to $H$, yields values which bear a striking similarity to those of the standard approach for this example. This may be interpreted as showing that complex resonances (in the standard sense) are very little affected by perturbations of the operator outside the potential barrier which gives rise to the resonance.

We now discuss two very general methods, the first real and the second complex, for computing the higher-order spectra, which are more appropriate for large matrices. Let $\mathscr{H}$ be any finite-dimensional Hilbert space, and let $z \rightarrow A(z)$ be an analytic function defined on a region $U \subseteq \mathbf{C}$, whose values are operators on $\mathscr{H}$, i.e. an analytic operator pencil. We define the spectrum of $A$ to be the set of $z \in U$ such that $A(z)$ is not invertible. Since this coincides with the set of zeros of the analytic function $p(z):=\operatorname{det}(A(z))$, it must be a discrete subset of $U$, excluding the uninteresting case in which $A(z)$ is singular for every $z \in U$. We describe a method for computing $\operatorname{Spec}(A)$ which does not involve the use of determinants.

If $(x, y) \in U$, we define $\sigma(x, y)$ to be the smallest singular value of the matrix $A(x+$ $i y)$. There are well-developed techniques for computing singular values, for example via reduction of the matrix to bidiagonal form, described in [31, Chapter 7]. 


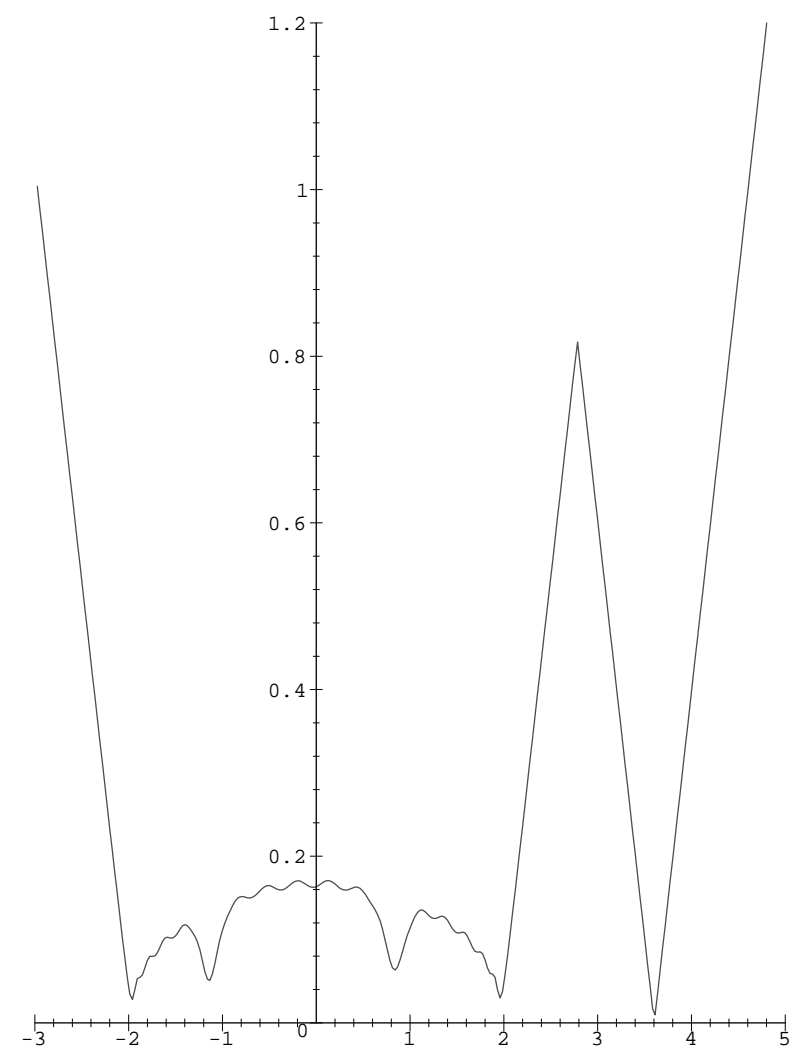

Figure 4: The graph of $F(s)$ for real $s$, with clear local minima near -1.14 and 0.84 and an eigenvalue near 3.60 . 


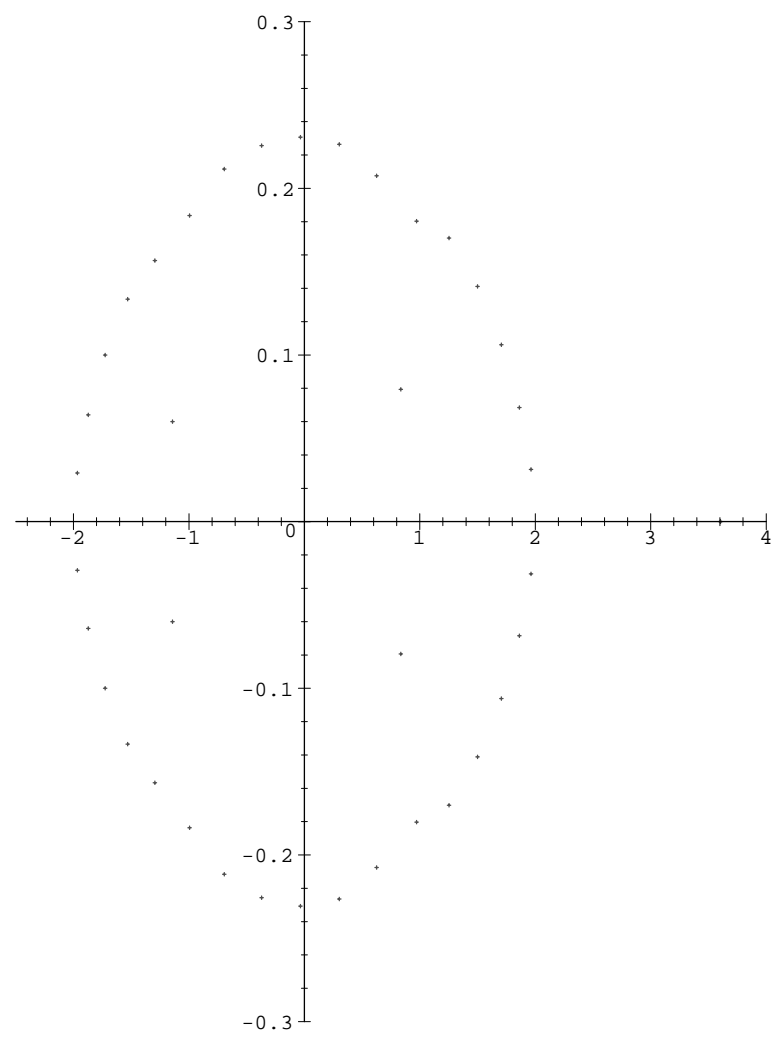

Figure 5: A plot of the complex resonances, as defined in this paper. 
It follows from the formula

$$
\sigma(x, y)=\inf \{\|A(z) \phi\| /\|\phi\|: 0 \neq \phi \in \mathscr{H}\}
$$

that $\sigma$ is a non-negative Lipschitz continuous function, and that it vanishes precisely at the points of $\operatorname{Spec}(A)$. One can now try to find the zeros of $\sigma$ by using a standard algorithm to move from an initial guess in steps towards a local minimum of $\sigma$. The following theorem guarantees that this procedure succeeds.

Theorem 21. Generically, the only local minima of the function $\sigma$ are the points at which it vanishes. Moreover, the function $\sigma(x, y)^{-2}$ is subharmonic on the set $V:=U \backslash \operatorname{Spec}(A)$.

Proof. Both statements follow by an analysis of the function $\rho:=\sigma^{-1}$ defined on $V$. We have

$$
\rho(x, y)=\left\|A(z)^{-1}\right\| .
$$

Given $c:=a+i b \in V$, suppose that $\phi, \psi$ are vectors of norm 1 such that

$$
\rho(a, b)=\left\langle\psi, A(z)^{-1} \phi\right\rangle
$$

for $z=c$. Because the RHS of the above equation is an analytic function, there exists $z$ in any neighbourhood of $c$, however small, such that

$$
\begin{aligned}
\rho(x, y) & \geqslant\left|\left\langle\psi, A(z)^{-1} \phi\right\rangle\right| \\
& >\left|\left\langle\psi, A(a+i b)^{-1} \phi\right\rangle\right| \\
& =\rho(a, b)
\end{aligned}
$$

unless the RHS is constant. The word "generically" in the theorem refers to the possibility that the RHS might be constant. While this is may indeed happen, it cannot do so if, for example, $U=\mathbf{C}$ and $\left\|A(z)^{-1}\right\| \rightarrow 0$ as $|z| \rightarrow \infty$.

Next let $\left\{u_{r}\right\}_{r=1}^{n}$ be an orthonormal basis of $\mathscr{H}$. Then

$$
\begin{aligned}
\rho(x, y)^{2} & =\left\|\left\{A(z)^{-1}\right\}^{*} A(z)^{-1}\right\| \\
& =\sup _{\|\phi\|=1} \sum_{r=1}^{n}\left|\left\langle u_{r}, A(z)^{-1} \phi\right\rangle\right|^{2} .
\end{aligned}
$$

The proof is completed by combining the invariance of the set of subharmonic functions under sums and suprema with the fact that $|g(z)|^{2}$ is subharmonic for every analytic function $g$.

Our second method of computing the complex resonances of an analytic operator pencil involves determining the smallest eigenvalue of $A(z)$ instead of its smallest singular value. We make the same assumptions on the operator-valued analytic function as in Theorem 21 . Given $z \in U$, we define $f(z)$ to be the smallest eigenvalue of $A(z)$ using the following lexicographic ordering of $\mathbf{C}$ : we put $r \mathrm{e}^{i \theta} \leqslant s \mathrm{e}^{i \phi}$ if $0 \leqslant r<s<\infty$, or if $r=s$ and $0 \leqslant \theta \leqslant \phi<2 \pi$.

Theorem 22. If a is a resonance of $A$ whose associated spectral projection has rank 1, then there exists $\delta>0$ such that $f$ is analytic on $\{z:|z-a|<\delta\}$ with $f(a)=0$.

Proof. If $\gamma$ is a small enough circle with centre 0 the contour integral

$$
P_{a}:=\frac{1}{2 \pi i} \int_{\gamma}(A(a)-w)^{-1} \mathrm{~d} w
$$


defines the spectral projection of $A(a)$ associated with its eigenvalue 0 . Replacing $a$ by $z$ in this formula the spectral projection $P_{z}$ is still of rank 1 and depends anaytically on $z$, provided that $z$ is close enough to $a$ by Kato's theory of analytic perturbations of the spectrum [20]. For such $z, A(z)$ has only one eigenvalue inside $\gamma$. This eigenvalue equals $f(z)$, and depends analytically on $z$.

Computationally, the application of this method involves evaluating the eigenvalues of $A(z)$ at some point which is suspected to be close to a complex resonance, and then using a modification of Newton's method for analytic functions to move towards a zero of $f$.

We have not attempted to write code to implement either of the above two ideas. While finding the smallest eigenvalue of a non-self-adjoint matrix is probably slower than finding its smallest singular value, the subsequent iterative procedure may well be faster because one can exploit the analyticity of the function concerned.

There is an extensive spectral theory [16], [26], [29] of polynomial operator pencils, including quadratic operator pencils of the form of (2.8). We refer to [30] and sources cited there for an account of the delicate issues involved in defining the spectrum of unbounded quadratic operator pencils, but confine ouselves to the bounded and finite-dimensional cases here. This theory suggests two other methods of finding the eigenvalues of the pencil. The first depends upon the existence of a factorisation

$$
N(z):=Q-2 z B+z^{2} I=\left(Z_{1}-z I\right)\left(Z_{2}-z I\right)
$$

for any square matrices $Q$ and $B$. Our particular choices of $Q$ and $B$ put us in the self-adjoint weakly coupled case, and we have $Z_{2}^{*}=Z_{1}$ and

$$
\operatorname{Spec}\left(Z_{1}\right) \subseteq\{z: \operatorname{Im}(z) \geqslant 0\}
$$

Given such a formula, it follows immediately that the set of eigenvalues of the quadratic pencil is just the union of the sets of eigenvalues of the two operators $Z_{1}$ and $Z_{2}$. For references to the literature on factorisations of this type, see [21], [16], [26], [29]. Unfortunately, existing procedures for obtaining $Z_{1}$ and $Z_{2}$ from $Q$ and $B$ are either indirect or not well adapted to efficient computation, so one cannot implement this approach.

The second method depends upon a theorem which may be extended to general polynomial operator pencils. It may often be the best method of calculating the spectrum of quadratic operator pencils, since it reduces the problem to standard eigenvalue estimates, the only cost being the doubling of the size of the matrices involved. For an implementation of this method see [12].

Theorem 23. Let $Q$ and $B$ be bounded operators on a Banach space $\mathcal{B}$, and let $z \in \mathbf{C}$. Then the operator $N(z):=Q-2 z B+z^{2} I$ is invertible if and only if the operator $T-z I$ acting on $\mathscr{B} \times \mathscr{B}$ is invertible, where

$$
T:=\left(\begin{array}{cc}
2 B & -Q \\
I & 0
\end{array}\right)
$$

Moreover, one then has

$$
(T-z I)^{-1}=\left(Q-2 z B+z^{2} I\right)^{-1}\left(\begin{array}{cc}
-z I & Q \\
-I & 2 B-z I
\end{array}\right) .
$$

Finally, $N(z)$ has a non-zero kernel if and only if $z$ is an eigenvalue of $T$. 
Proof. All of the statements are obtained by direct computations. If $\mathscr{B}$ is finite-dimensional, almost all of the statements are consequences of the identity

$$
\operatorname{det}(T-z I)=\operatorname{det}\left(Q-2 z B+z^{2} I\right) .
$$

\section{Appendix A. Maple programs}

The Maple programs in [9], [10], [11], [12] are provided as an electronic appendix to this paper. The appendix is available to subscribers to the journal at:

http://www.lms.ac.uk/jcm/1/lms97005/appendix-a/.

\section{References}

1. S. Agmon. Proc. St. Jeans de Monts Conf. (1996) . 62

2. S. Agmon, 'On perturbations of embedded eigenvalues', Partial differential equations and mathematical physics, the Danish-Swedish Analysis Seminar, 1995 (ed. A Melin and L Hörmander, Birkhauser, Boston, Basel, Berlin, 1996) pp. 1-14. 62

3. W. Arveson. Apple Macintosh programs available at ftp://math.berkeley.edu/pub/Preprints/Bill_Arveson/Mac_programs http://math.berkeley.edu/ arveson/. 43, 56

4. W. Arveson, ' $C^{*}$-algebras and numerical linear algebra', J. Functional Anal. 122 (1994) 333-360. 43, 44, 55

5. W. Arveson, 'The role of $C^{*}$-algebras in infinite-dimensional numerical linear algebra', Contemp. Math. 167 (1994) 115-129. 43, 55

6. J. Avron, P. H. M. van Mouche and B. Simon, 'On the measure of the spectrum for the almost Mathieu operator', Commun. Math. Phys. 132 (1990) 103-118. Erratum, Commun. Math. Phys. 139 (1991) 215. 43, 57, 57

7. F. Chatelin, Spectral approximations of linear operators (Academic Press, New York, 1983). 45

8. H. L. Cycon, R. G. Froese, W. Kirsch and B. Simon, Schrödinger operators, with applications to quantum mechanics and global geometry. Texts and Monographs in Physics (Springer-Verlag, 1987). 62, 62

9. E. B. DAviES. Maple V.4 program eigen 35 available at http://www.lms.ac.uk/jcm/1/lms97005/appendix-a/. 45, 46, 52, 72

10. E. B. DAviES. Maple V.4 program mathieu11 available at http://www.lms.ac.uk/jcm/1/lms97005/appendix-a/. 57, 72

11. E. B. DAvies. Maple V.4 program polyn 1 available at http://www.lms.ac.uk/jcm/1/lms97005/appendix-a/. 63, 63, 63, 72

12. E. B. DAvies. Maple V.4 program reson 25 available at http://www.lms.ac.uk/jcm/1/lms97005/appendix-a/. 67, 71, 72

13. E. B. DAviEs, Spectral theory and differential operators (Cambridge Univ. Press, 1995). 44

14. P. A. Deift and R. Hempel, 'On the existence of eigenvalues of the Schrödinger operator $h-\lambda w$ in a gap of $\sigma(h)^{\prime}$, Commun. Math. Phys. (1986) 461-490. 44 
15. F. Gesztesy, G. M. Graf and B. Simon, 'The ground state energy of Schrödinger operators', Commun. Math. Phys. 150 (1992) 375-384. 57

16. I. Gohberg, P. LANCASTER and L. RoDMAn, Matrices and indefinite scalar product; operator theory and applications 8 (Birkhäuser Verlag, Basel, Boston, Stuttgart, 1983). 71, 71

17. E. M. Harrell II, 'Generalizations of Temple's inequality', Proc. Amer. Math. Soc. 69 (1978) 271-276. 42

18. E. M. Harrell II, 'General lower bounds for resonances in one dimension', Commun. Math. Phys. 86 (1982) 221-225. 63

19. T. Kato, 'On the upper and lower bounds of eigenvalues', J. Phys. Soc. Japan 4 (1949) 334-339. 42

20. T. KATO, Perturbation theory of linear operators (Springer-Verlag, Berlin, Heidelberg, New York, 1966). 48, 66, 71

21. M. G. Krein and G. K. LANGER, 'On some mathematical principles of the linear theory of damped oscillations of continua', English translation, Integral Eqns. Oper. Theory 1 (1978) 364-399, 539-566. 71

22. A. LAPtEV and Y. SAFARov, 'A generalization of the Berezin-Lieb inequality', Transl. Math. Monogr. (Amer. Math. Soc., Providence, RI, 1996) (2) 175, pp. 69-79. 53

23. A. Laptev and Y. Safarov, 'Szegő type limit theorems', J. Funct. Anal. 138 (1996) 544-559. 54, 54

24. Y. LASt, 'Amost everything about the Almost Mathieu operator I', Proc. XIth Intern. Congress of Math. Phys., Paris (1994) . 57, 57

25. Y. LAST, 'Zero measure spectrum for the Almost Mathieu operator', Commun. Math. Phys. 164 (1994) 421-432. 57, 57

26. A. S. MARKus, Introduction to the spectral theory of polynomial operator pencils, Transl. Math Monogr. (Amer. Math. Soc., Providence, RI, 1988). 71, 71

27. U. Mertins, 'On the convergence of the Goerisch method for self-adjoint eigenvalue problems with arbitrary spectrum', Zeit. für Anal. und ihre Anwendungen, J. for Analysis and its Applications 15 (1996) 661-686. 42, 44

28. M. Plum, 'Guaranteed numerical bounds for eigenvalues', Spectral Theory and Computational Methods of Sturm-Liouville Problems, preprint, Lecture Notes in Pure and Applied Mathematics Series, 191 (eds Don Hinton and Philip W. ScHAEFER, Marcel Dekker, Inc., 1997). 44

29. M. Rosenblum and J. RovnJAK, 'The factorisation problem for non-negative operator pencils', Bull. Amer. Math. Soc. 77 (1971) 287-318. 71, 71

30. A. A. ShKalikov, 'Operator pencils arising in elasticity and hydrodynamics: the instability index formula', Operator theory: advances and applications 87 (Birkhäuser Verlag, Basel, Boston, Stuttgart, 1996), pp. 258-285. 71

31. D. S. WATKINS, Fundamentals of matrix computations (Wiley, 1991). 57, 67

32. H. WidOM, Toeplitz operators (Prentice-Hall, 1965). 44

33. S. Zimmerman and U. Mertins, 'Variational bounds to eigenvalues of self-adjoint eigenvalue problems with arbitrary spectrum', Zeit. für Anal. und ihre Anwendungen. J. for Analysis and its Applications 14 (1995) 327-345. 42, 44, 50 
E.B. Davies E.Brian.Davies@kcl.ac.uk

Department of Mathematics

King's College London

Strand

London WC2R 2LS 